\title{
Recent results on CP Violation in Charm sector by $\mathrm{LHCb}$
}

XVIII International Conference on Hadron Spectroscopy and Structure (HADRON 2019)

Guilin, China, 2019/08/16-21

\author{
SAUR Miroslav
}

(On behalf of the LHCb collaboration)

University of Chinese Academy of Sciences 


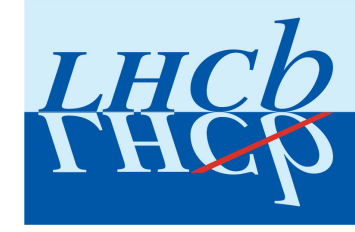

\section{LHCb detector and data}




\section{LHCb detector 2010-2018}

\section{LHCb
THC:}

$\rightarrow$ Single-arm forward spectrometer focused on heavy flavor $(b, c)$ physics

$\rightarrow$ Effective as multi-purpose detector in forward region

Vertex Locator(vertex reconstruction) Tracking system(particle reconstruction)

- Impact parameter resolution:

- $\epsilon$ (Tracking) $\sim 96 \%$

IJMPA 30 (2015) 1530022

\section{$20 \mu \mathrm{m}$}

- Decay time resolution 45 fs $\left(\tau_{\mathrm{B}} \sim 1.5 \mathrm{ps}\right)$

- $\delta p / p \sim 0.5 \%-1 \%(5-200 \mathrm{GeV})$

- $\sigma\left(m_{B \rightarrow h h}\right) \approx 22 \mathrm{MeV}$

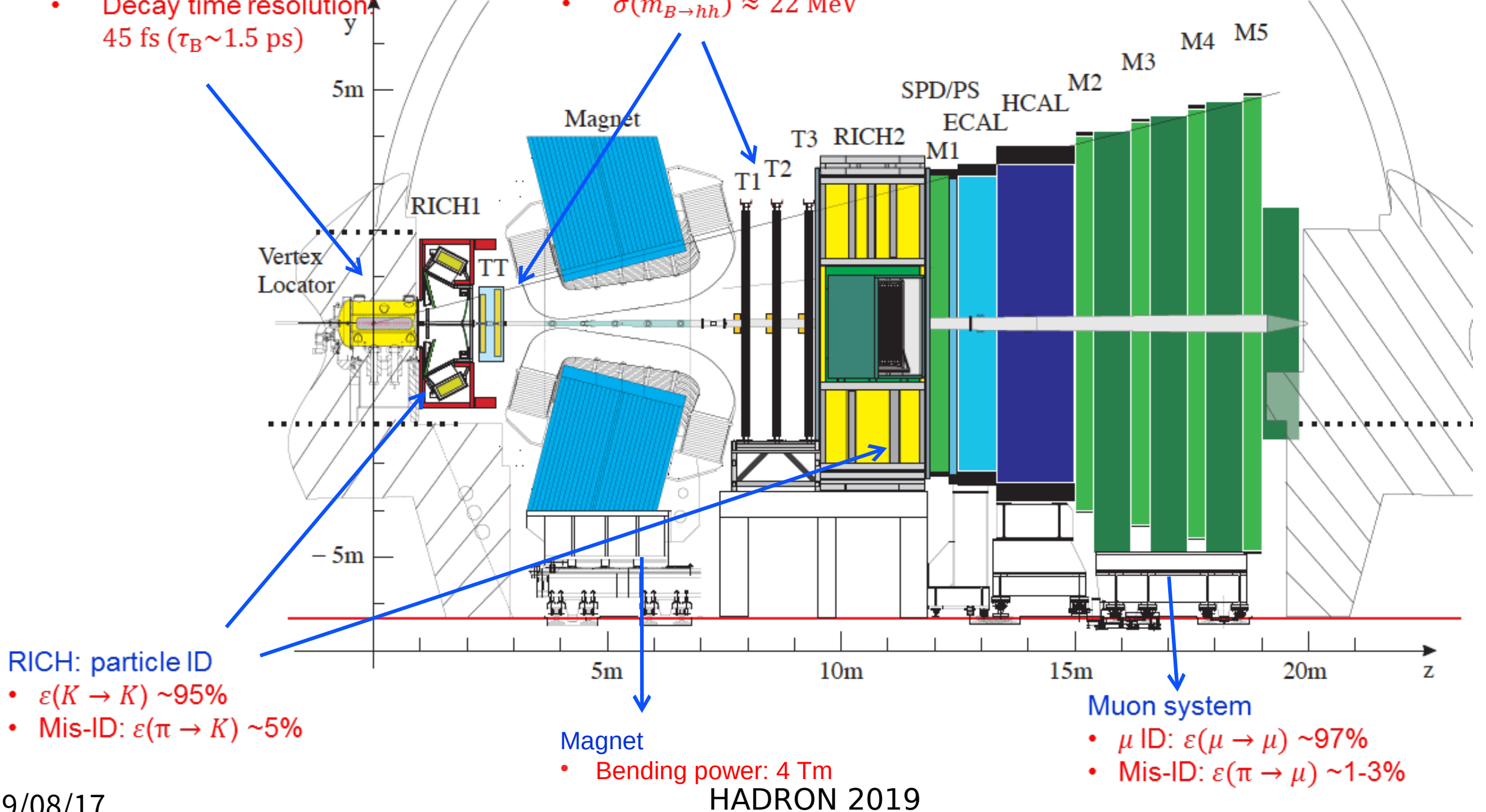




\section{Data taking 2010-2018}

$\rightarrow$ Run I (7-8 TeV, 2010-2012) and Run II (13 TeV, 2015-2018)

$\rightarrow$ Average efficiency of the data taking $>90 \%$

$\rightarrow$ Various systems: pp, p-Pb, Pb-Pb, SMOG (fixed target)

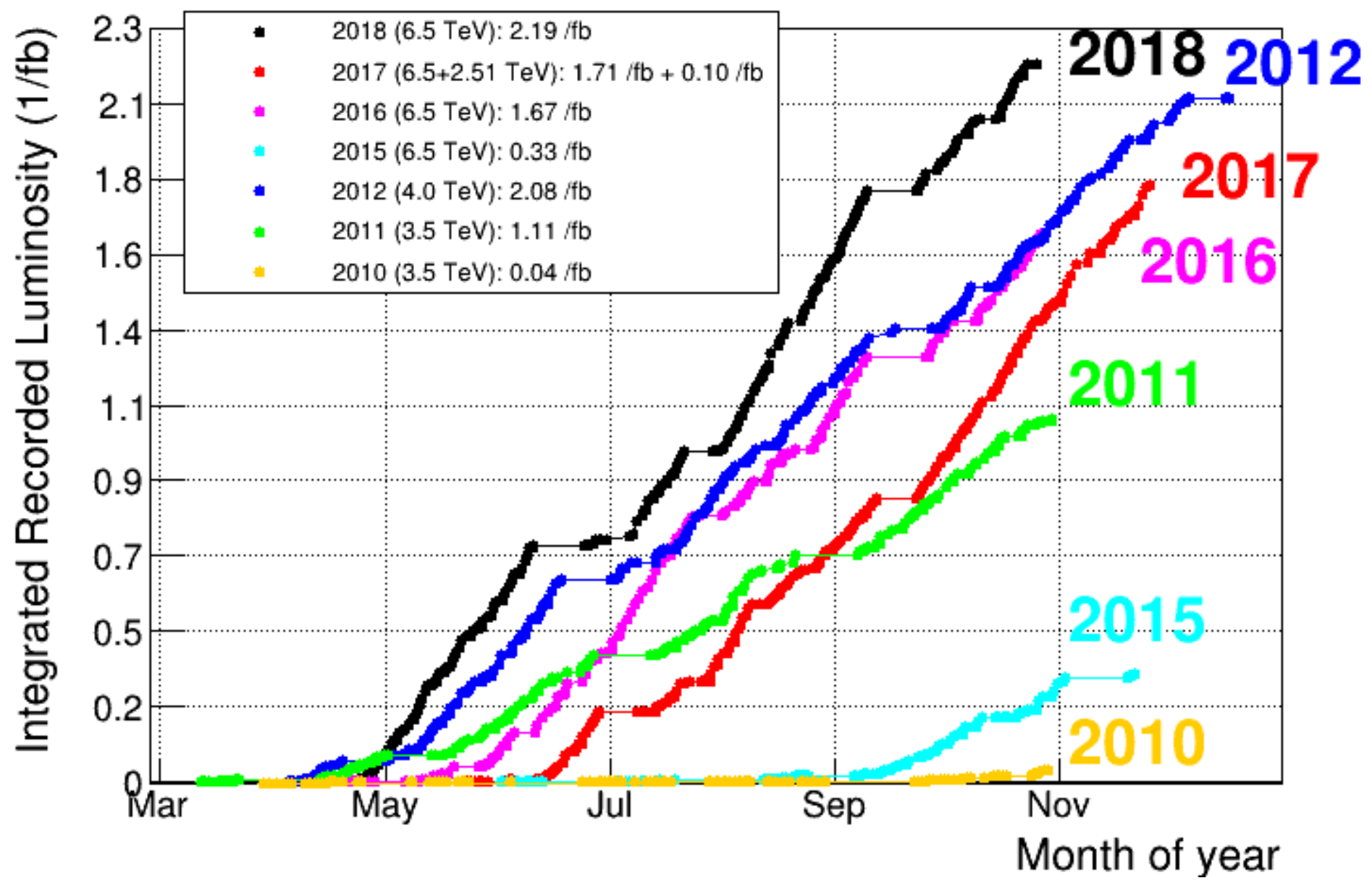




\section{Measurement of charm at LHCb}

$\rightarrow$ Large charm cross section at LHCb:

NPB 8711 (2016), JHEP05 074 (2017)

$$
\sigma(p p \rightarrow c \bar{c})=\begin{aligned}
& {[1419 \pm 12 \text { (stat.) } \pm 116 \text { (syst.) } \pm 65 \text { (frag.) }] \mu \mathrm{b} @ 7 \mathrm{TeV}} \\
& {[2369 \pm 3 \text { (stat.) } \pm 152 \text { (syst.) } \pm 118 \text { (frag.) }] \mu \mathrm{b} @ 13 \mathrm{TeV}}
\end{aligned}
$$

$\rightarrow$ Significant statistics collected already during the Run I:

$$
p_{\mathrm{T}}<8 G e V / c, 2.0<y<4.5
$$

- About $5 \times 10^{12} \mathrm{D}^{0}$ and $2 \times 10^{12} \mathrm{D}^{*+}$ collected

$\rightarrow$ Run II: higher collision energy and improved trigger $\rightarrow$ more statistics than Run I

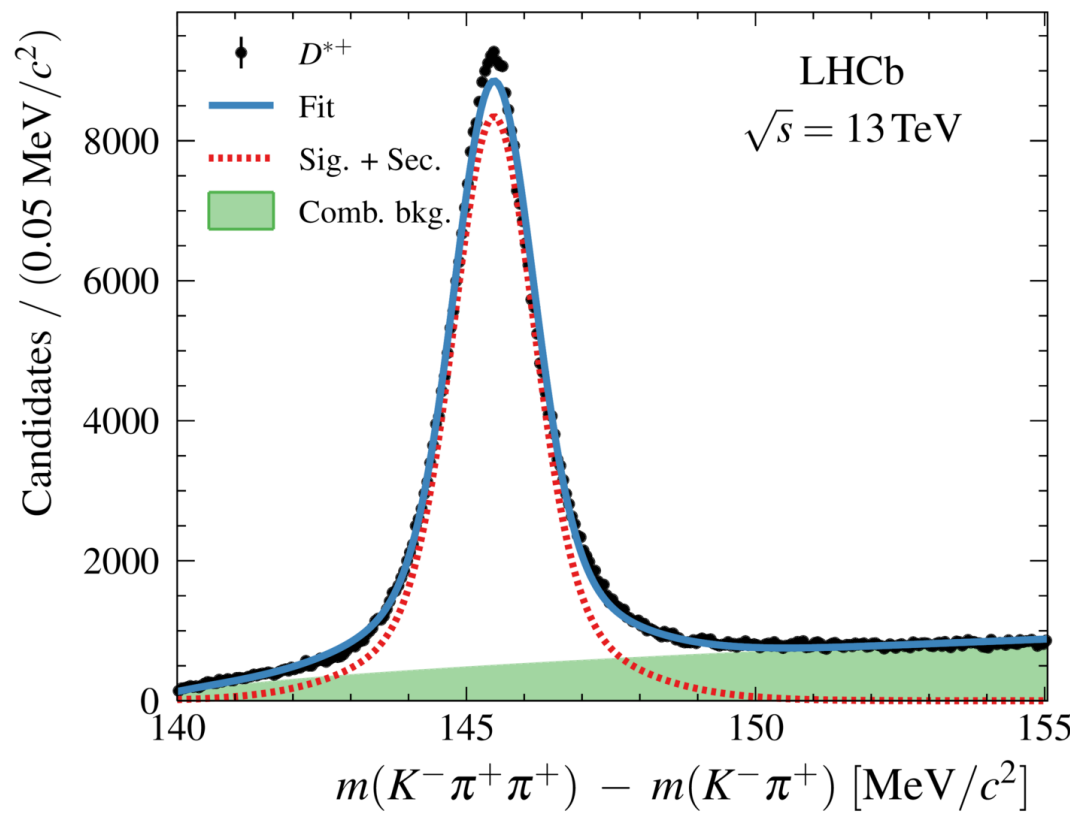

2019/08/17

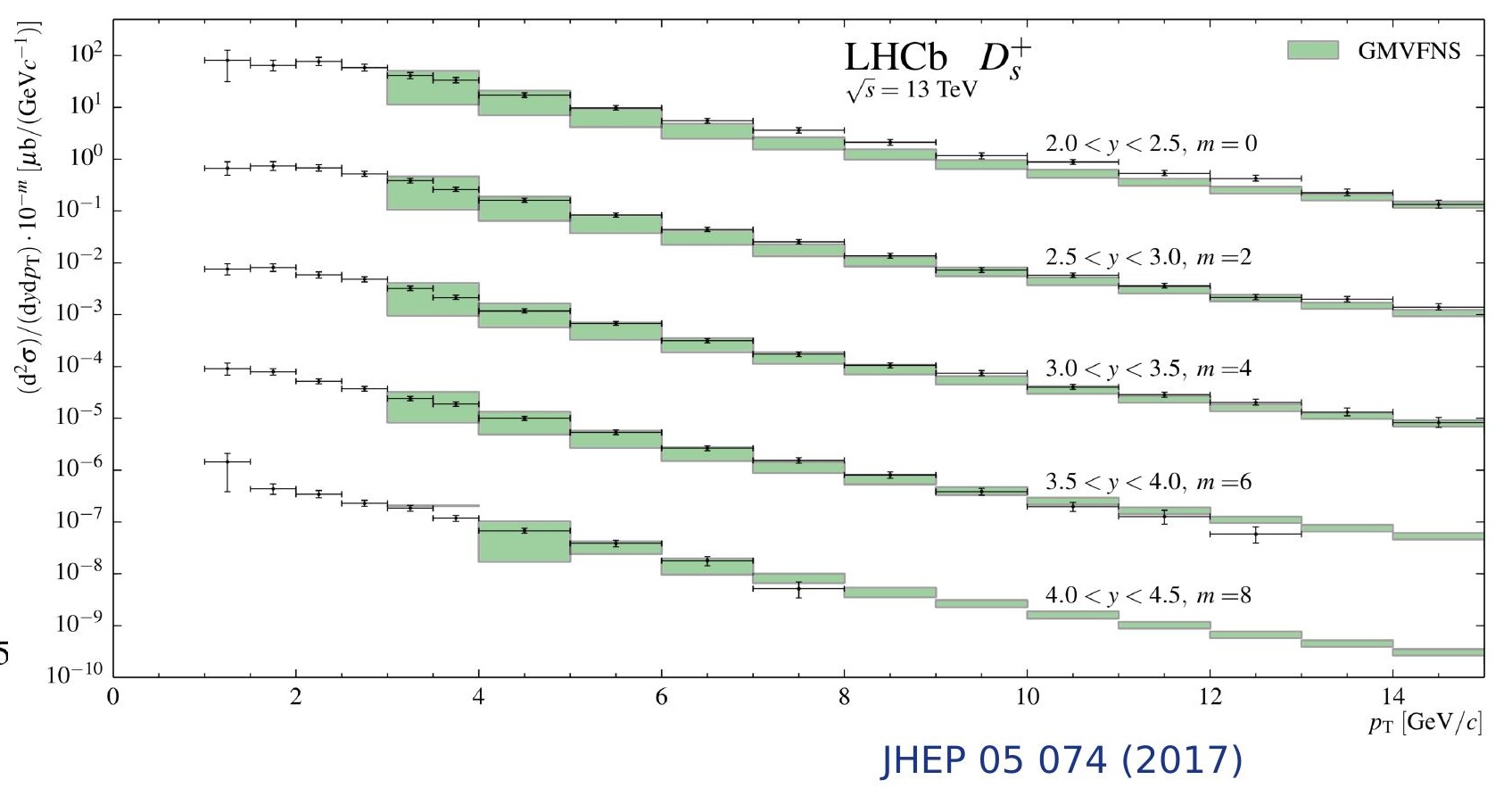

HADRON 2019 


\section{Experimental aspects at $\mathrm{LHCb}$}

$\rightarrow$ Flavor tagging: prompt vs secondary $\rightarrow$ LHCb uses both methods
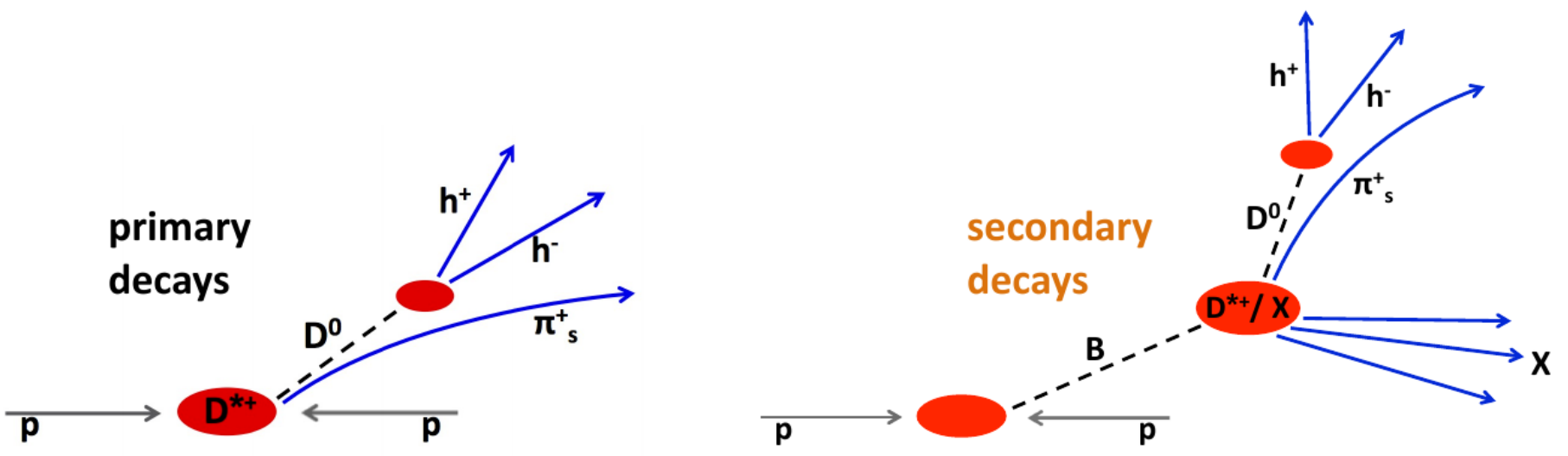

$\rightarrow$ Production asymmetries (charge dependent):

- Different cross-section for $\mathrm{D}_{(\mathrm{s})}^{+} / \mathrm{D}_{(\mathrm{s})}^{-}, \Lambda_{\mathrm{c}}^{+} / \Lambda_{\mathrm{c}}^{-}, \ldots$

$\rightarrow$ Detection asymmetries (charge and momentum dependent):

- Different interactions with the detector material ( $\mathrm{K}^{+}$vs $\mathrm{K}^{-}, \pi^{+}$vs $\pi^{-}$)
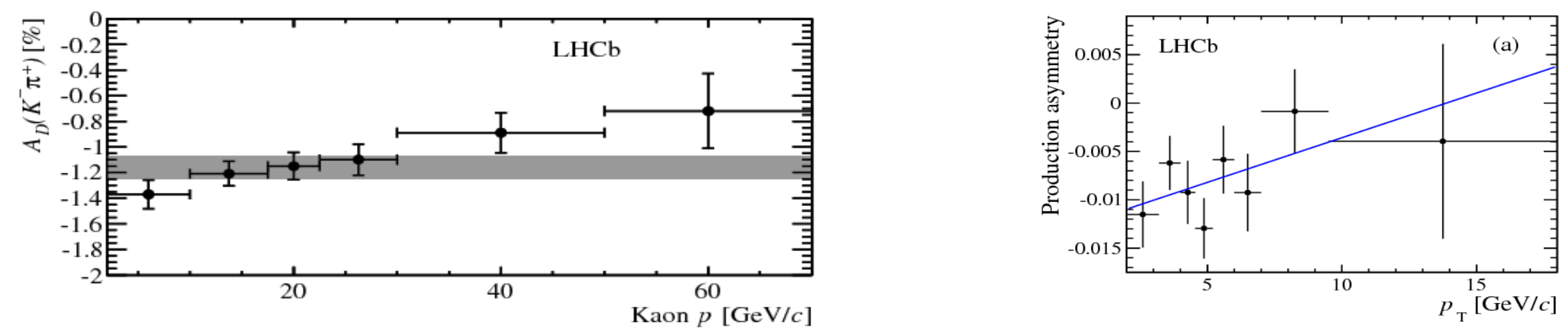

HADRON 2019

PLB 718902 (2013) 


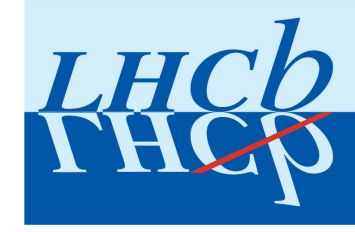

\section{Charge-Parity Violation}




\section{Mixing of $\mathrm{D}^{0}-\overline{\mathbf{D}}^{0}$}

\section{LHCD}

$\rightarrow \mathrm{D}^{0}$ mesons are produced as a flavor eigenstates, but decays as mass eigenstates $\mathrm{D}_{1}$ and $\mathrm{D}_{2}:\left|\mathrm{D}_{1}\right\rangle=p\left|\mathrm{D}^{0}\right\rangle+q\left|\overline{\mathrm{D}}^{0}\right\rangle,\left|\mathrm{D}_{2}\right\rangle=p\left|\mathrm{D}^{0}\right\rangle-q\left|\overline{\mathrm{D}}^{0}\right\rangle, \quad|q|^{2}+|p|^{2}=1$

$\rightarrow$ Mixing occurs in the case: $\Delta M=M_{1}-M_{2} \neq 0$ or $\Delta \Gamma=\Gamma_{1}-\Gamma_{2} \neq 0$

$\rightarrow$ Associated mixing parameters: $x=\frac{\Delta M}{\Gamma}, y=\frac{\Delta \Gamma}{2 \Gamma}$, where: $\Gamma=\frac{\Gamma_{1}+\Gamma_{2}}{2}$

$\rightarrow$ Influence of short and long distance effects

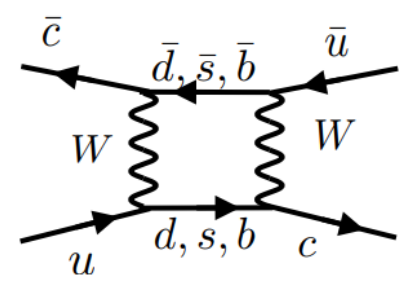

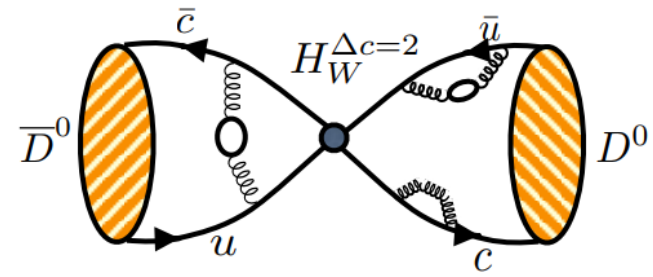

short distance

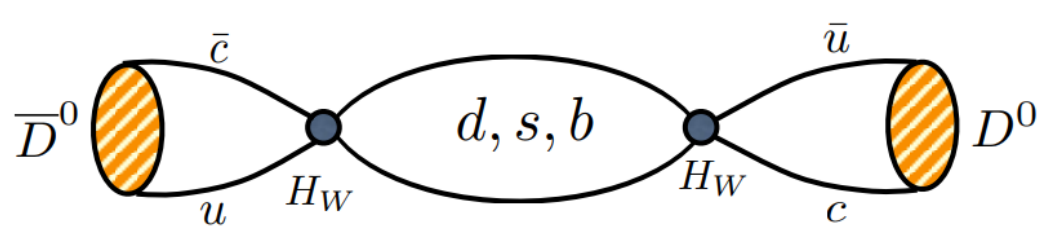

long distance

$\rightarrow$ For the small mixing parameters $\left(x, y<10^{-2}\right)$ the time-dependent asymmetry can be approximated as:

$$
A_{C P}(t) \equiv \frac{\Gamma\left(D^{0}(t) \rightarrow f\right)-\Gamma\left(\bar{D}^{0}(t) \rightarrow f\right)}{\Gamma\left(D^{0}(t) \rightarrow f\right)+\Gamma\left(\bar{D}^{0}(t) \rightarrow f\right)} \simeq A_{C P}^{d i r, f}-A_{\Gamma} \frac{f}{t_{D}}
$$

where $A_{\Gamma}$ is the asymmetry between effective decay widths of $\mathrm{D}^{0}$ and $\overline{\mathrm{D}}^{0}$

$$
A_{\Gamma}=\frac{\Gamma\left(D^{0} \rightarrow f\right)-\Gamma\left(\bar{D}^{0} \rightarrow f\right)}{\Gamma\left(D^{0} \rightarrow f\right)+\Gamma\left(\bar{D}^{0} \rightarrow f\right)}
$$




\section{CPV classification}

$\rightarrow$ CPV is present in the SM via Cabibbo-Kobayashi-Maskawa (CKM) mechanism, but is too weak to explain the Baryon asymmetry of the Universe

$\rightarrow$ CPV has been firstly observed at Strange [PRL 13138 (1964)] and Bottom [NP A 675 (2000), 398-403] sector, first observation in Charm sector (PRL 122 (2019) 211803)

$\rightarrow$ Two types of CPV: Indirect (CPV in mixing, CPV in interference) and Direct

\section{CPV in mixing}

$\rightarrow$ Independent on final state

$\rightarrow$ Different mixing rates $\mathrm{D}^{0} \rightarrow \overline{\mathrm{D}}^{0}$ and $\overline{\mathrm{D}}^{0} \rightarrow \mathrm{D}^{0}$ $\left|\frac{q}{p}\right| \neq 1$

$\rightarrow$ Accessible via the using flavor specifics decays

$\rightarrow$ SM prediction: $\mathcal{O}\left(10^{-4}\right)$
CPV in interference

$\rightarrow$ Possibility of interference between mixing and decay amplitudes

$$
\phi=\arg \left(\frac{q \bar{A}_{f}}{p A_{f}}\right)
$$

$\rightarrow$ Can be observed as a decay-time-dependent difference in decay rates and as a timeintegrated difference

$\rightarrow$ SM prediction: $\mathcal{O}\left(10^{-4}\right)$

\section{Direct CPV}

$\rightarrow$ Only possible CPV for charged hadrons

$\rightarrow$ Occurs in the case:

$$
\left|\frac{\bar{A}_{\bar{f}}}{A_{f}}\right| \neq 1
$$

$\rightarrow$ Typically (for SCS modes): $A_{C P}<10^{-4}-10^{-3}$ 


\section{Charm sector and CPV}

$\rightarrow$ Charm is unique, gives sensitivity to new physics coupling to up-type quark

$\rightarrow$ Charm is also difficult for theory calculations

$\rightarrow$ Complementary to direct searches for BSM particles

$\rightarrow$ BSM contributions could be hidden in loops

$\rightarrow$ Assuming generic BSM scenarios, much larger scale are accessible with respect to direct searches

$\rightarrow$ Flavour physics and CPV lead to breakthrough in particle physics many times

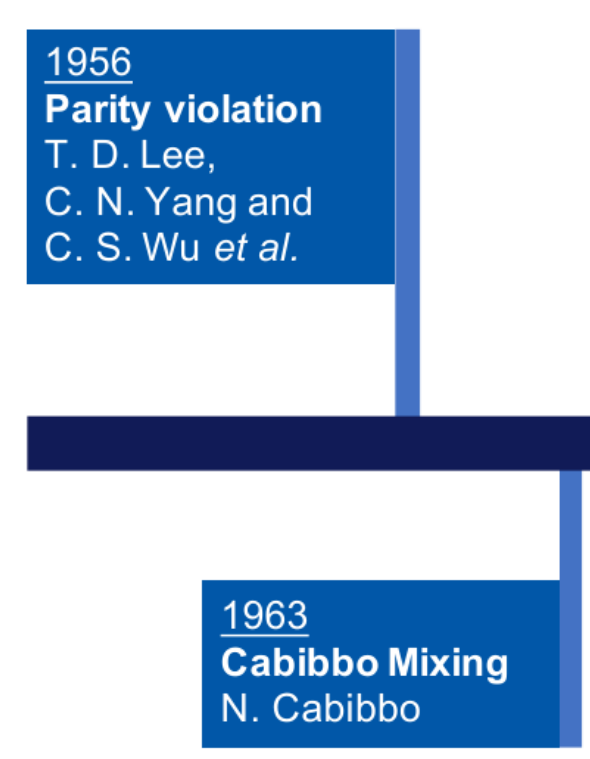

$\frac{1963}{\text { Cabibbo Mixing }}$

N. Cabibbo

$\frac{1964}{\text { Strange particles: }}$
CP violation in $K$
meson decays
J. W. Cronin,
V. L. Fitch et al.

\section{3}

The CKM matrix

M. Kobayashi and

T. Maskawa

\section{$\underline{2001}$}

Beauty particles:

$C P$ violation in $B^{0}$

meson decays

BaBar and Belle

collaborations 


\section{Recent LHCb results on CP violation in Charm}

1. Measurement of the mass difference between neutral charm-meson eigenstates (PHYS. REV. LETT. 122 (2019) 231802)

2. Search for time-dependent $C P$ violation in $D^{0} \rightarrow K^{+} K^{-}$ and $D^{0} \rightarrow \pi^{+} \pi^{-}$decays (LHCB-CONF-2019-001; INSPIRE: 1735332)

3. Observation of $C P$ violation in charm decays (PHYS. REV. LETT. 122 (2019) 211803) 
1. Measurement of the mass difference between neutral charm-meson eigenstates (PHYS. REV. LETT. 122 (2019) 231802) 


\section{$\mathrm{D}^{0}$ mass eigenstates $\Delta \mathrm{m}$ : introductiof}

$\rightarrow C P V$ is an interference effect

$\rightarrow$ How to enhance our sensitivity?

$\rightarrow$ LHCb Run I full sample

$\rightarrow$ Prompt and semileptonic production of $\mathrm{D}^{0} \rightarrow \mathrm{K}_{\mathrm{S}}^{0} \pi^{+} \pi^{-}$

$\rightarrow$ Around $1.3 \times 10^{6}$ signal candidates for prompt production and around $1.0 \times 10^{6}$ for semileptonic decays

$\rightarrow$ Channel with a rich resonance spectrum

$\rightarrow$ Good sensitivity to mixing and timedependent CPV parameters via varying strong phases

$\rightarrow$ Experimentally complicated (decay dynamics and acceptance effects)

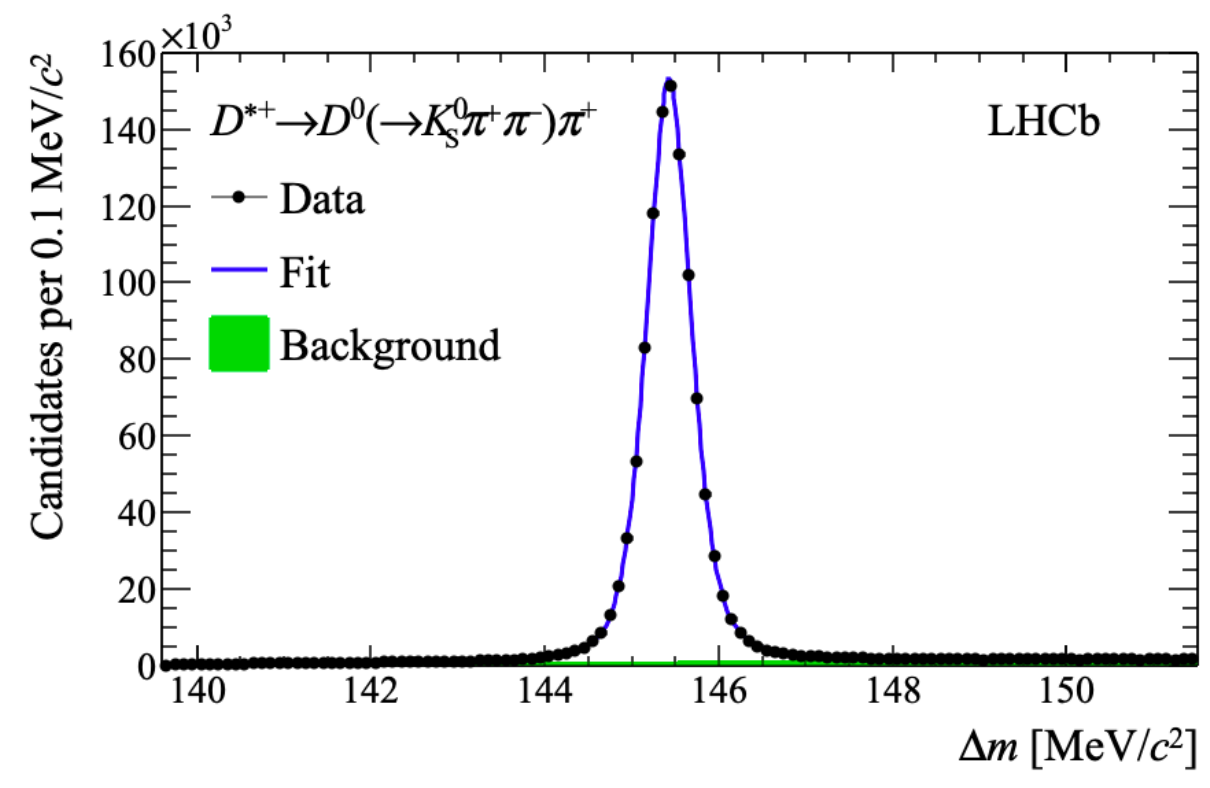

PHYS. REV. LETT. 122 (2019) 231802

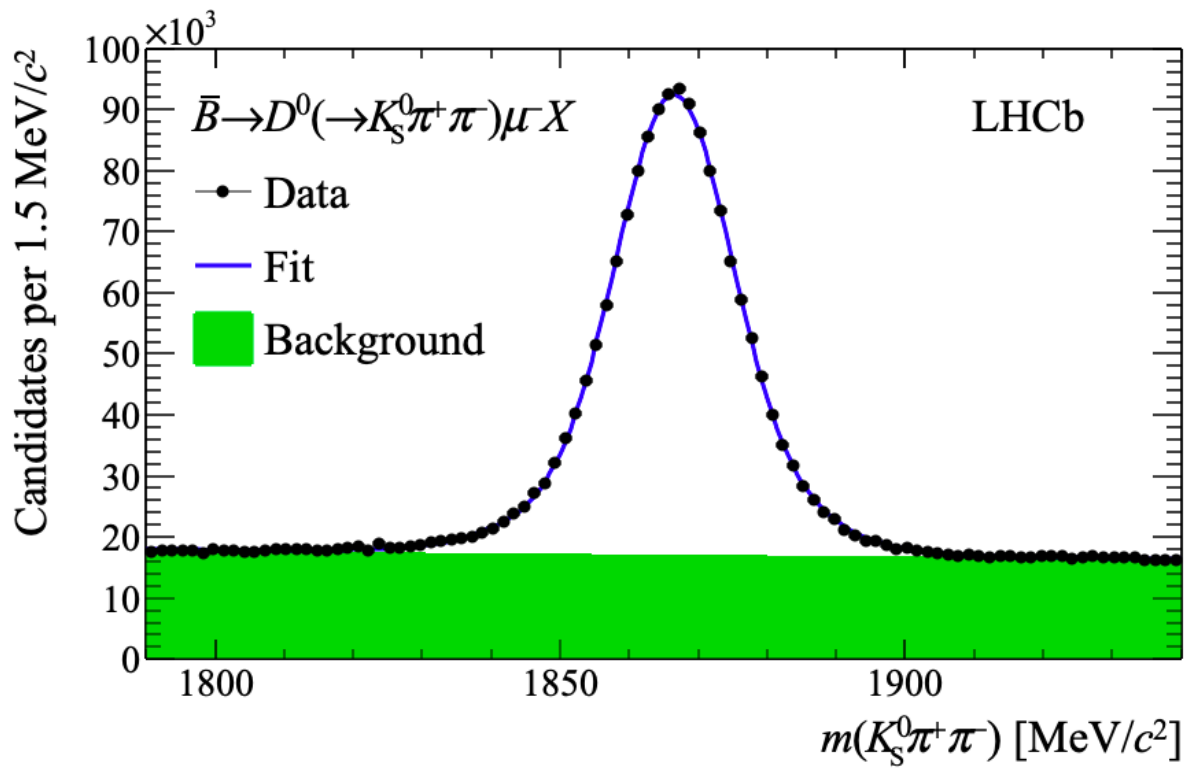




\section{$D^{0}$ mass eigenstates $\Delta \mathrm{m}$ : method}

$\rightarrow$ Bin flip method (Phys. Rev. D 99, 012007): a novel approach minimizing dependence on amplitude model and detector acceptance

$\rightarrow$ Data are binned in Dalitz plane $\left(R_{1-8}^{+/-}\right)$to keep strong phases approximately constant; input is taken from CLEO (Phys. Rev. D 82, 112006)

$\rightarrow$ Data are also binned in decay time (20 bins)

$\rightarrow$ Ratio of yields in opposition bins across the bisection is measured

- Cancellation of acceptance effects, also a good sensitivity to $x$

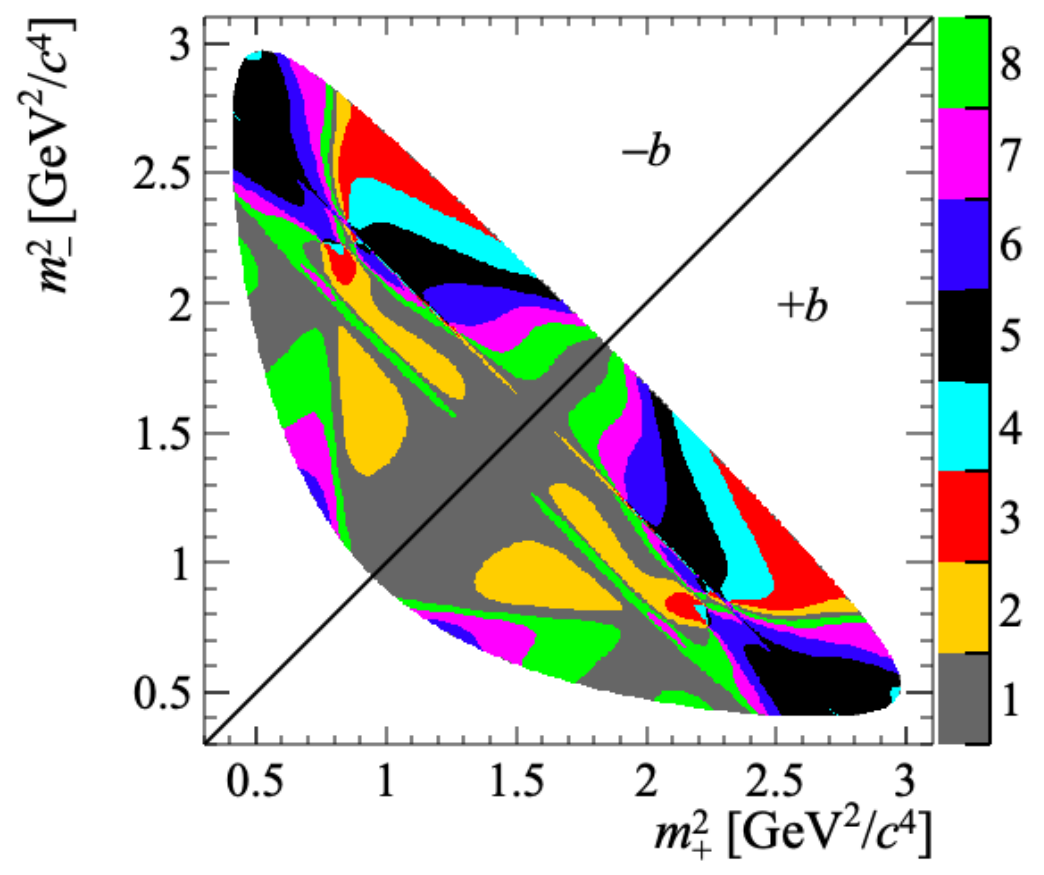

PHYS. REV. LETT. 122 (2019) 231802

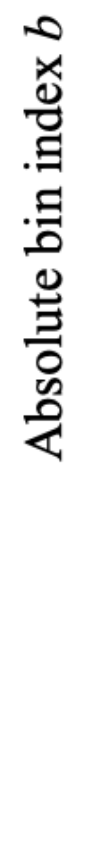

HADRON 2019

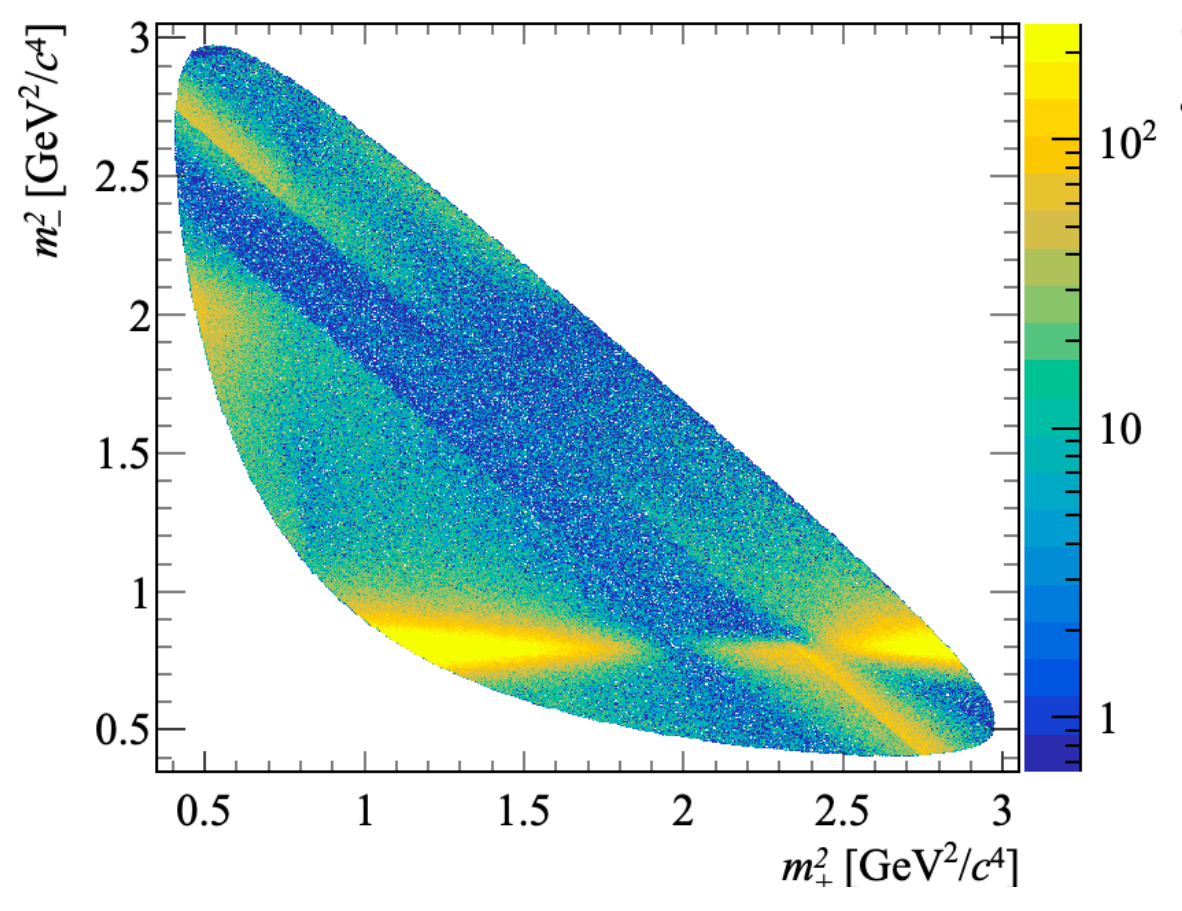

$14 / 30$ 


\section{$D^{0}$ mass eigenstates $\Delta \mathrm{m}$ : fits}

$\rightarrow$ Simultaneous least-squares fit* to prompt and semileptonic data

$\rightarrow$ Offset due to sample-specific efficiency variations across Dalitz plot

$\rightarrow C P$-averaged yield ratios as function of $t / \tau$

$\rightarrow$ Mixing measurement

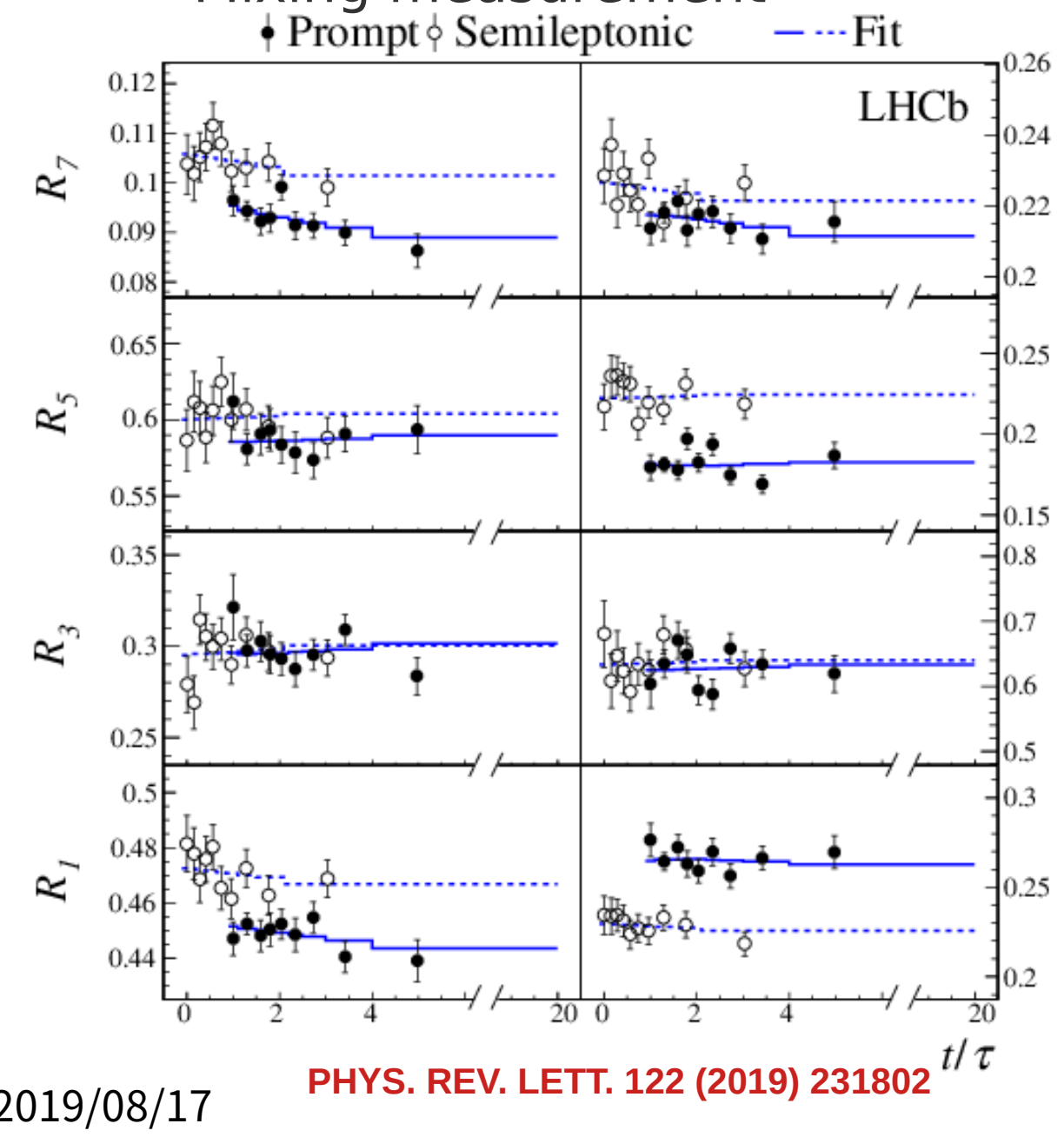

$\rightarrow$ Differences of $\mathrm{D}^{0}$ and anti-D $\mathrm{D}^{0}$ yield ratios as function of $t / \tau$

$\rightarrow$ Search for CP violation

$$
\text { - Prompt } \phi \text { Semileptonic - - F Fit }
$$

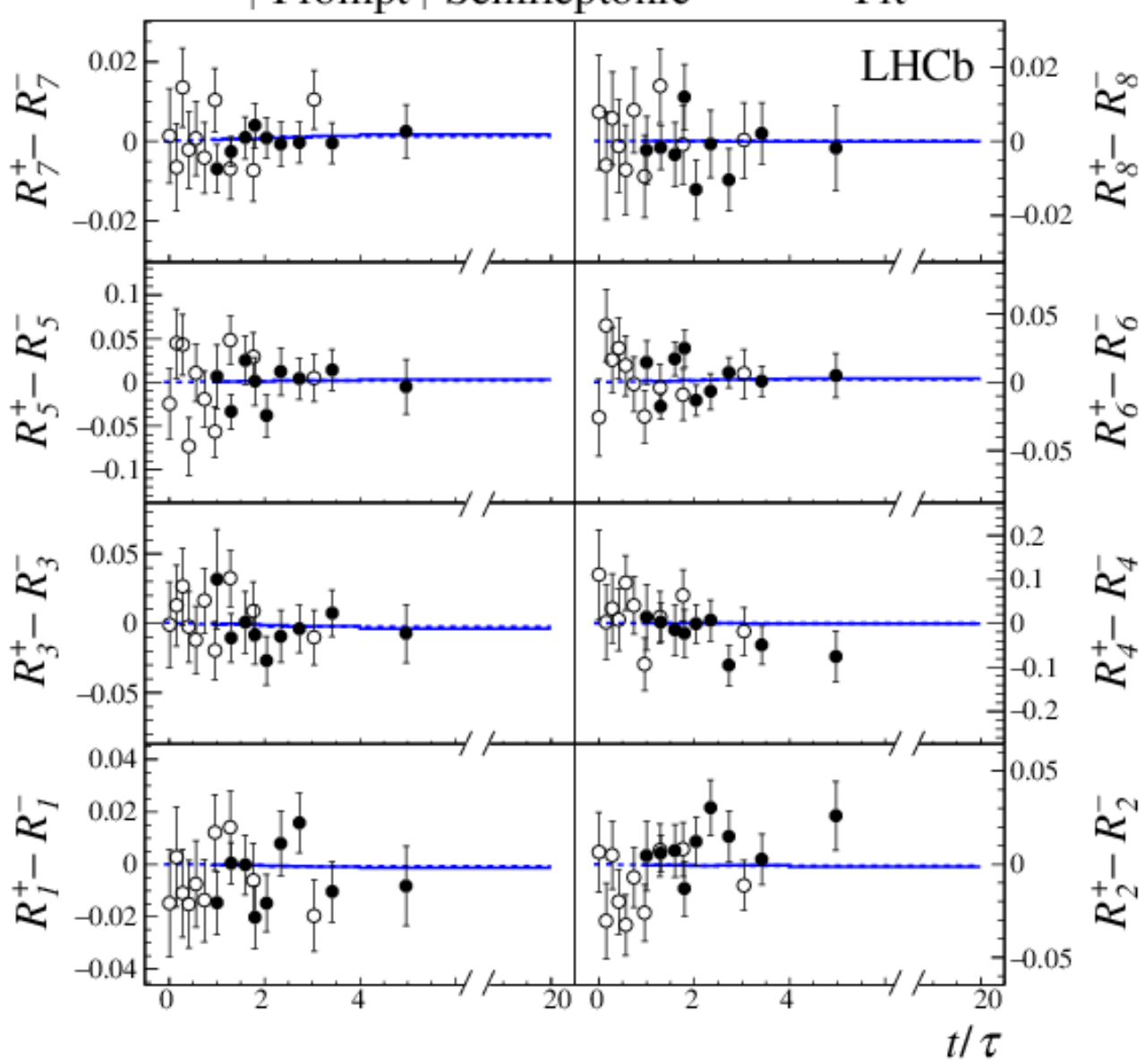

HADRON 2019 details in backup slide 40

\section{LHCD}

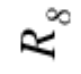

20

2

$\approx$ 


\section{$\mathrm{D}^{0}$ mass eigenstates $\Delta \mathrm{m}$ : results}

$\rightarrow$ The most precise measurement of $x$ done by a single experiment, consistent with CP symmetry scenario

\begin{tabular}{lcc}
\hline Parameter & Value & $95.5 \%$ CL interval \\
\hline$x\left[10^{-2}\right]$ & $0.27_{-0.15}^{+0.17}$ & {$[-0.05,0.60]$} \\
$y\left[10^{-2}\right]$ & $0.74 \pm 0.37$ & {$[0.00,1.50]$} \\
$|q / p|$ & $1.05_{-0.17}^{+0.22}$ & {$[0.55,2.15]$} \\
$\phi$ & $-0.09_{-0.16}^{+0.11}$ & {$[-0.73,0.29]$} \\
\hline
\end{tabular}

$\rightarrow$ Combined with the world average, first evidence of $x>0$ larger than $3 \sigma$
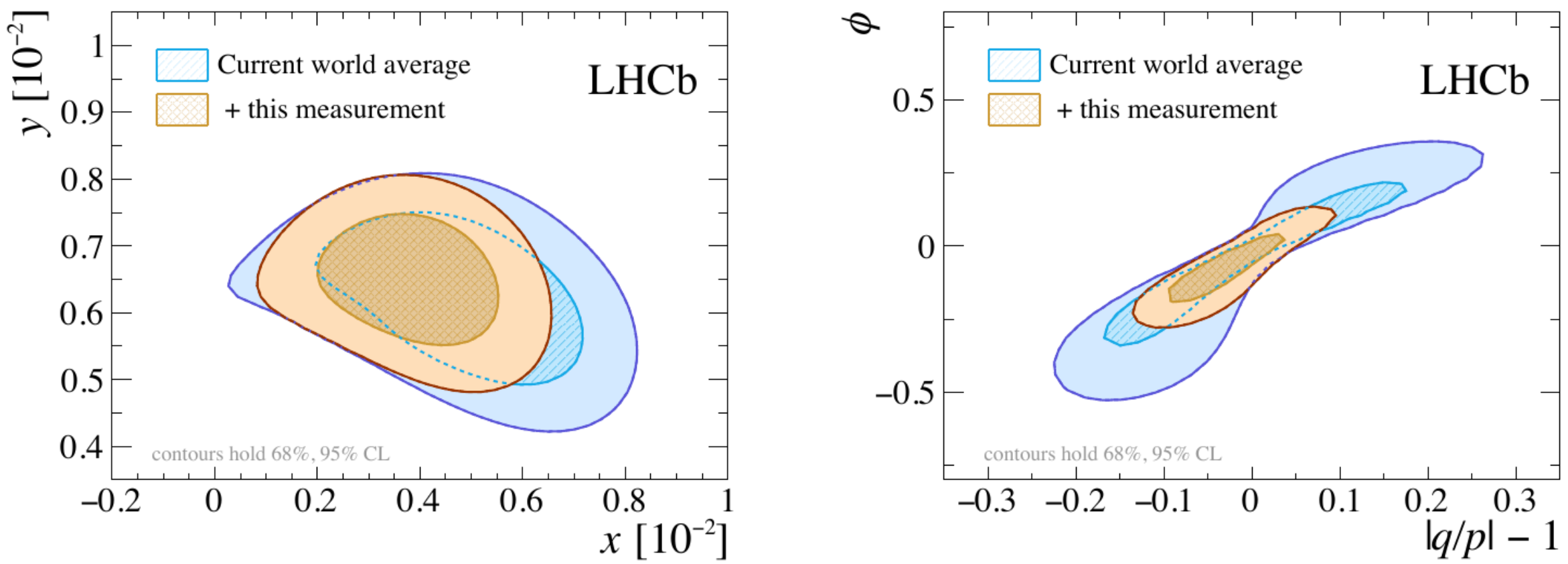
2. Search for time-dependent $C P$ violation in $D^{0} \rightarrow K^{+} K^{-}$ and $D^{0} \rightarrow \pi^{+} \pi^{-}$decays (LHCB-CONF-2019-001; INSPIRE: 1735332) 


\section{$C P V(t)$ in $D^{0} \rightarrow h^{+} h:$ introduction}

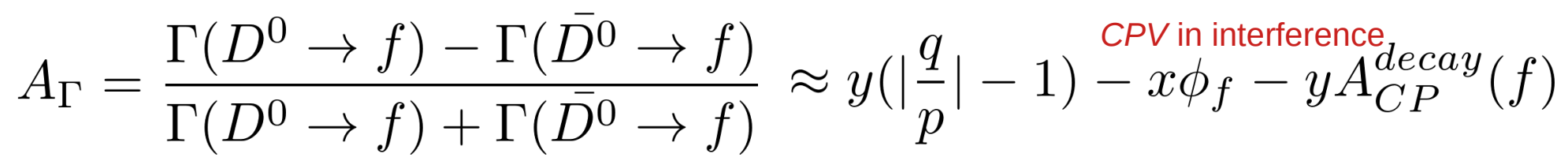

$$
\begin{aligned}
& C P V \text { in mixing }
\end{aligned}
$$

$\rightarrow$ LHCb 2015-2016 data, prompt $D^{*+}$ decays utilized for a tagging of D0 decays

$\rightarrow$ Analysis done using two signal channels $\mathrm{D}^{0} \rightarrow \mathrm{K}^{+} \mathrm{K}^{-} / \pi^{+} \pi^{-}\left(17 \times 10^{6} / 5 \times 10^{6}\right)$

$\rightarrow \mathrm{D}^{0} \rightarrow \mathrm{K}^{-} \pi^{+}$control channel $\left(146 \times 10^{6}\right)$ used for a full analysis procedure
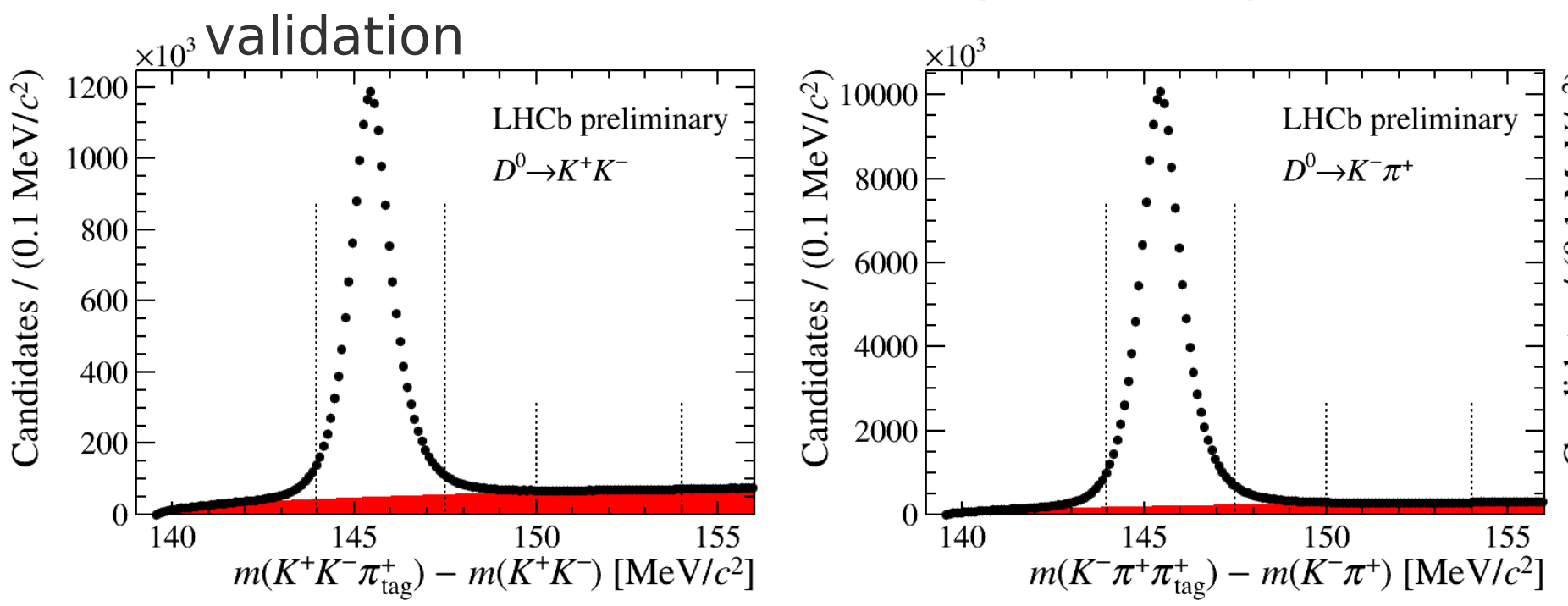

$\rightarrow$ Asymmetry measured in 21 decay time bins

$\rightarrow$ Current world average, $(-3.2 \pm 2.6) \times 10^{-4}$, dominated by LHCb Run I measurement (Phys. Rev. Lett. 118, 261803)

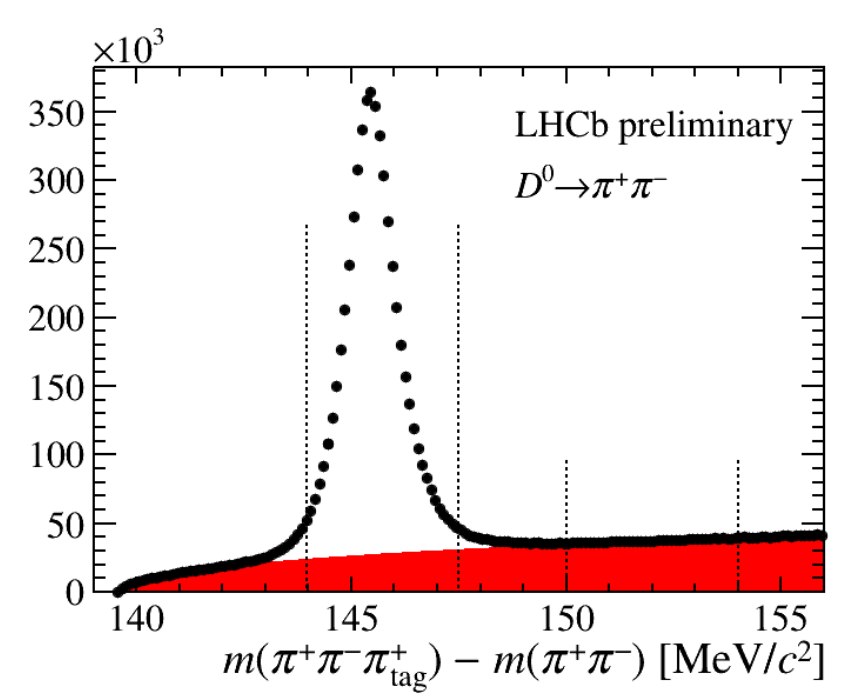

LHCb-CONF-2019-001

$18 / 30$ 


\section{$C P V(t)$ in $D^{0} \rightarrow h^{+} h$ : detector asymm}

$\rightarrow$ Time and momentum-dependent asymmetries arise from two main sources

- Momentum-dependent detection asymmetry from tagging pion

- Correlation between the measured decay time and the momentum of the $\mathrm{D}^{0}$ due to trigger requirements

$\rightarrow$ Effect can be cancelled by weighting events between $\mathrm{D}^{0}$ and anti-D ${ }^{0}$ candidates

- Separate weighting for different experimental conditions (magnet polarity, year)

- 3D momentum weighting

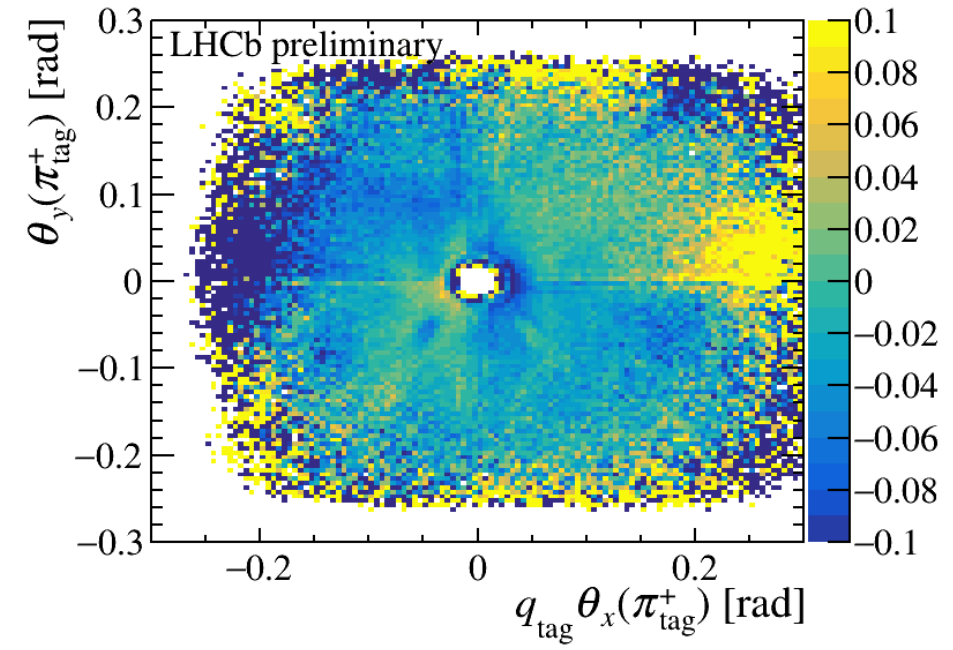

2019/08/17

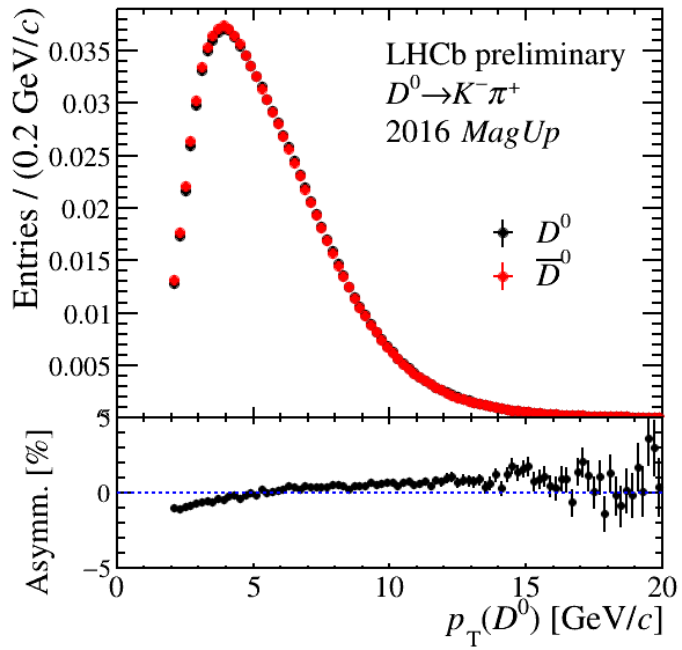

HADRON 2019
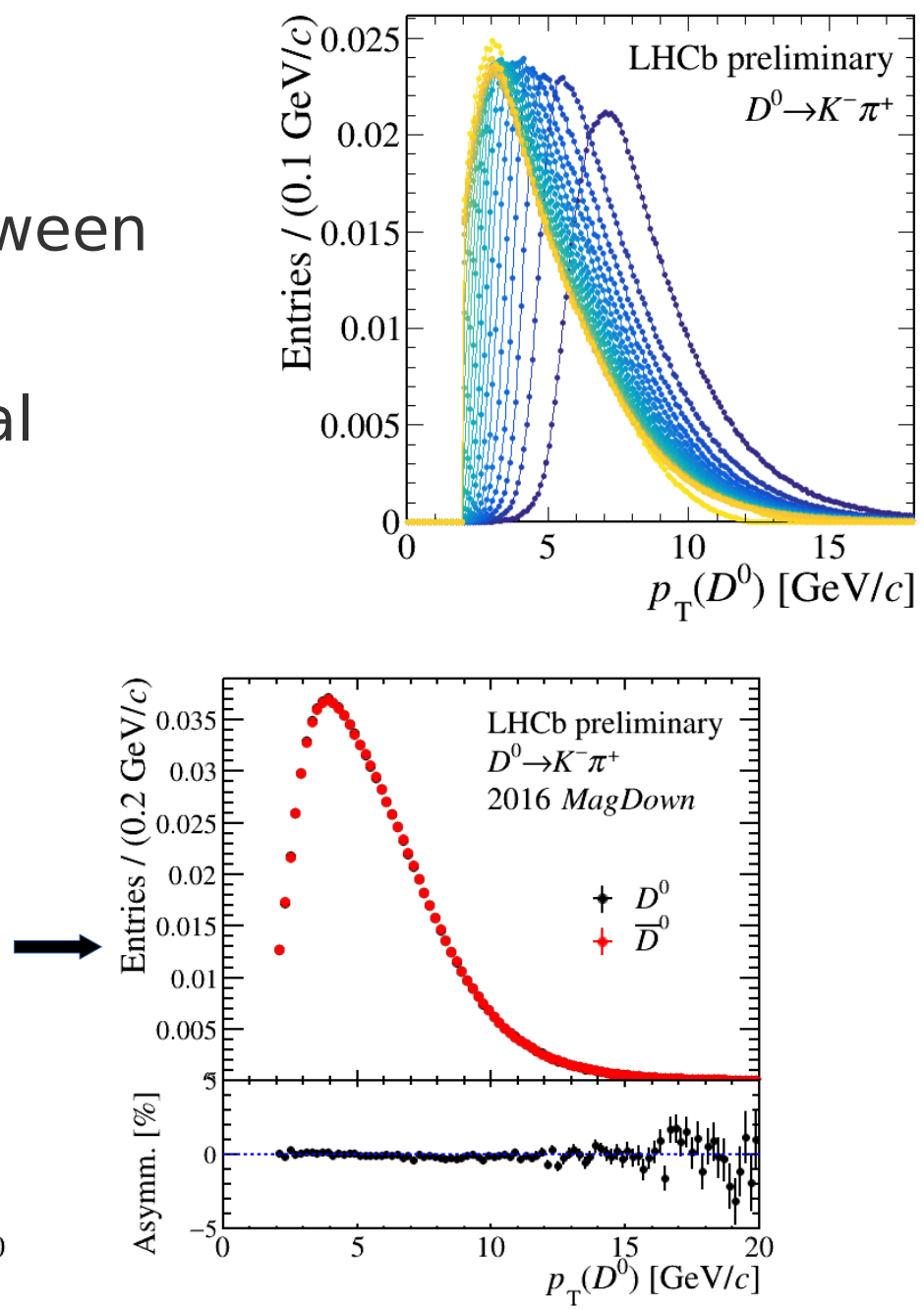

LHCb-CONF-2019-001 


\section{$C P V(t)$ in $D^{0} \rightarrow h^{+} h^{-}$: systematic}

\section{LHCD}

$\rightarrow$ Contamination of $\mathrm{D}^{*+}$ by the secondary decays

- Measured decay time of secondary decays biased to longer decay time

- Fraction of secondary decays increases as a function of time

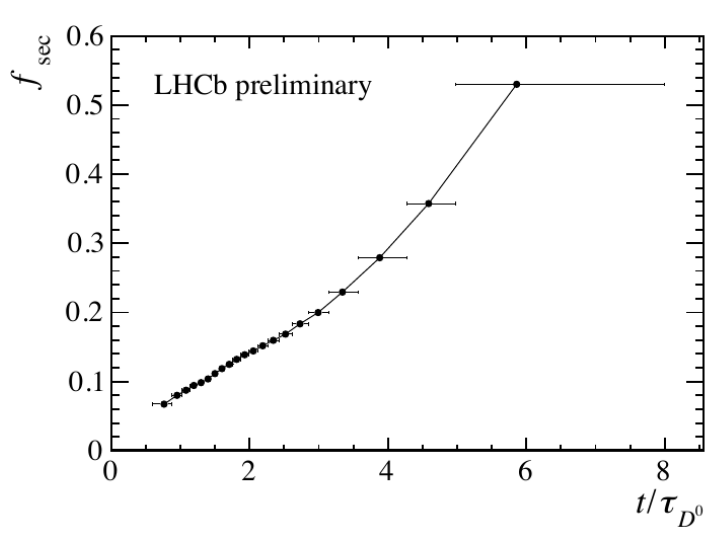

$\rightarrow$ Kinematic weighting depends on the exact binning

- Bins has be to kept large enough to avoid large statistical fluctuations

- Control channel used for bin size optimization

\begin{tabular}{lcc}
\hline Source & $A_{\Gamma}\left(D^{0} \rightarrow K^{+} K^{-}\right)$ & $A_{\Gamma}\left(D^{0} \rightarrow \pi^{+} \pi^{-}\right)$ \\
\hline Secondary decays & 0.4 & 0.4 \\
$\Delta m$ background & 0.3 & 0.5 \\
$m\left(h^{+} h^{-}\right)$background & 0.3 & 0.2 \\
Kinematic weighting & 0.3 & 0.3 \\
\hline Sum in quadrature & 0.7 & 0.8 \\
\hline
\end{tabular}


3. Observation of CP violation in charm decays (PHYS. REV. LETT. 122 (2019) 211803) 


\section{CPV in Charm: introduction}

$\rightarrow$ Full LHCb Run II data set

$\rightarrow \mathrm{D}^{0} \rightarrow \mathrm{K}^{+} \mathrm{K}^{-} / \pi^{+} \pi^{-}$decays

$\rightarrow$ Prompt $\left(44 \times 10^{6} / 13 \times 10^{6}\right)$ and semileptonic production

$\left(9 \times 10^{6} / 3 \times 10^{6}\right)$

$\rightarrow$ Using Turbo data stream online reconstruction of data (Comput. Phys. Commun. 208 (2016) 35)

$\rightarrow$ Fit to invariant mass distribution to extract the raw asymmetries

$\rightarrow$ However, raw asymmetries are influenced by the production and detection asymmetries
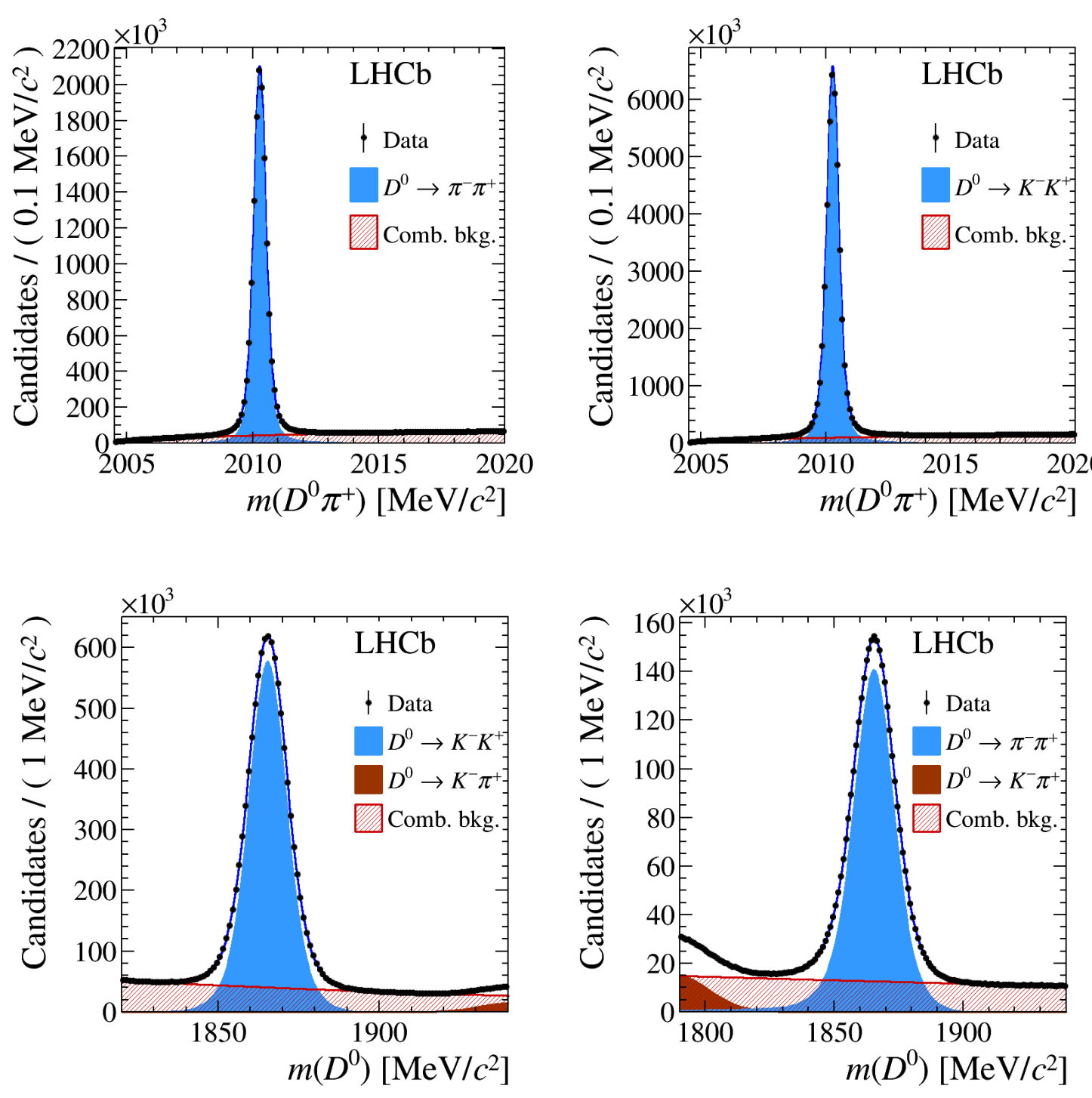

PHYS. REV. LETT. 122 (2019) 211803 


\section{$C P V$ in Charm: experimental issues}

$\rightarrow$ Detection and production asymmetries can be cancelled using suitable experimental procedure for prompt / semileptonic decays:

$$
\mathrm{A}_{\text {raw }}(\mathrm{f}) \approx \mathrm{A}_{\mathrm{CP}}(\mathrm{f})+\mathrm{A}_{\mathrm{D}}\left(\mathrm{D}^{0}\right)+\mathrm{A}_{\mathrm{D}}(\pi / \mu)+\mathrm{A}_{\mathrm{P}}\left(\mathrm{D}^{*+} / \mathrm{B}\right)
$$

$\rightarrow \quad A_{\text {raw }}(f)=\frac{N\left(D^{0} \rightarrow f\right)-N\left(\overline{D^{0}} \rightarrow f\right)}{N\left(D^{0} \rightarrow f\right)+N\left(\overline{D^{0}} \rightarrow f\right)}$ - experimentally accessible asymmetry

$\rightarrow \mathrm{A}_{\mathrm{CP}}(\mathrm{f})$ - physical CP asymmetry of final state $f$

$\rightarrow A_{D}\left(D^{0}\right)-D^{0}$ detection asymmetry, cancelled due to symmetric final states

$\rightarrow \mathrm{A}_{\mathrm{D}}(\pi / \mu)$ - detection asymmetry of tagging particle

$\rightarrow \mathrm{A}_{\mathrm{P}}\left(\mathrm{D}^{*+} / \mathrm{B}\right)$ - production asymmetry of mother particle

$\rightarrow$ Under the assumption of small experimental asymmetries, CP can be obtained as

$$
\Delta \mathrm{A}_{\mathrm{CP}} \equiv \mathrm{A}_{\mathrm{CP}}\left(\mathrm{K}^{+} \mathrm{K}^{-}\right)-\mathrm{A}_{\mathrm{CP}}\left(\pi^{+} \pi^{-}\right)=\mathrm{A}_{\mathrm{raw}}\left(\mathrm{K}^{+} \mathrm{K}^{-}\right)-\mathrm{A}_{\mathrm{raw}}\left(\pi^{+} \pi^{-}\right)
$$




\section{$C P V$ in Charm: fiducial selection}

$\rightarrow$ Due to LHCb geometry, low momentum particle can be kicked out from the detector acceptance

$\rightarrow$ Such a regions of phase space generate very large raw detector asymmetries

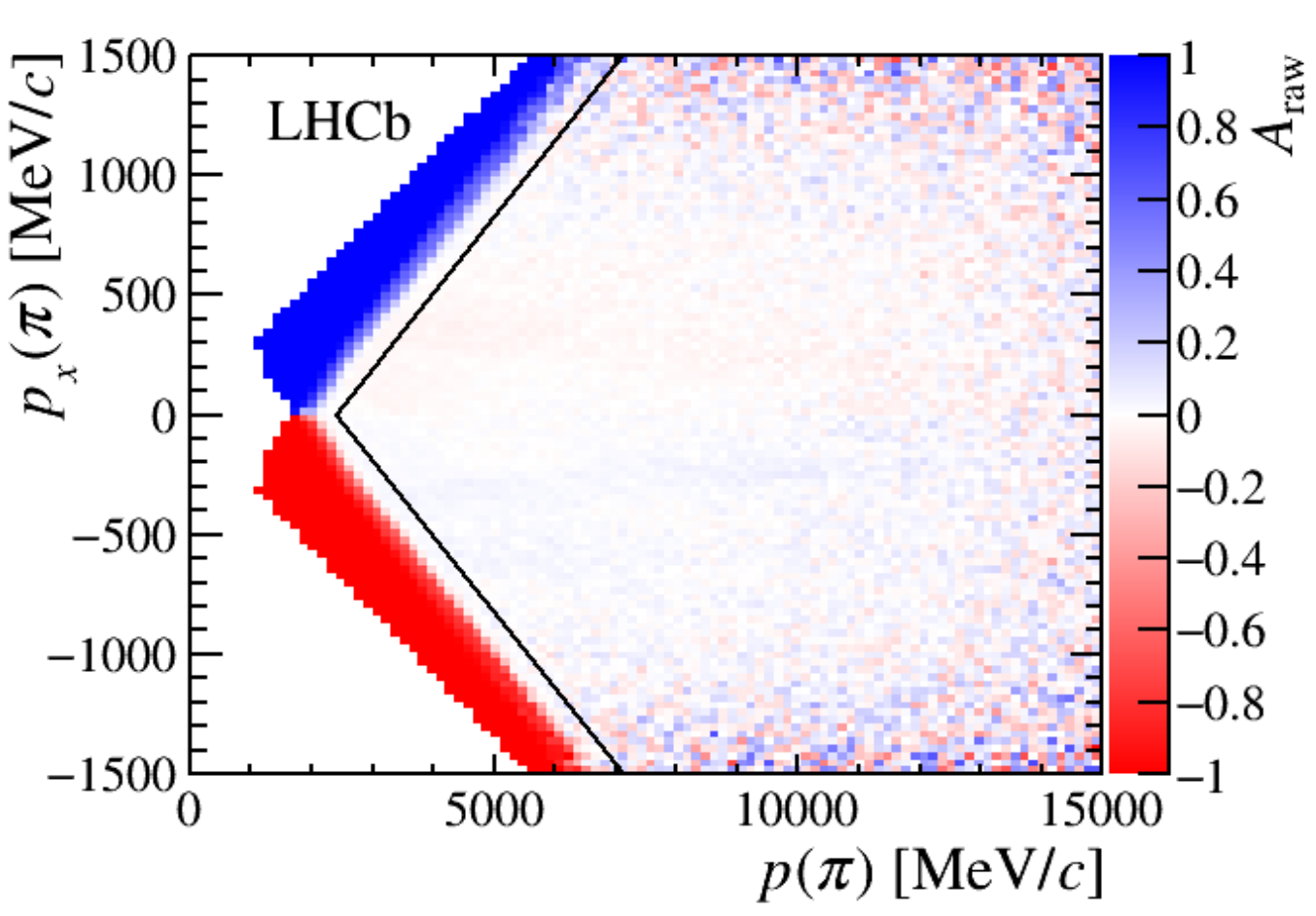

PHYS. REV. LETT. 122 (2019) 211803

$\rightarrow$ This part of phase space must be removed in order of kinematic equalization

$\rightarrow$ Same procedure for prompt/semileptonic decays $(\pi / \mu)$ 


\section{CPV in Charm: kinematic weighting MHEP}

$\rightarrow$ Detection and production asymmetries depend on the kinematic of the reconstructed particles

$\rightarrow$ Weighting procedure between modes to assure same kinematic

$\rightarrow$ Variables prompt/semileptonic: $p_{\mathrm{T}}\left(\mathrm{D}^{*}\right), p\left(\mathrm{D}^{*}\right), \phi\left(\mathrm{D}^{*}\right) / p_{\mathrm{T}}\left(\mathrm{D}^{0}\right), p\left(\mathrm{D}^{0}\right), \phi\left(\mathrm{D}^{0}\right)$
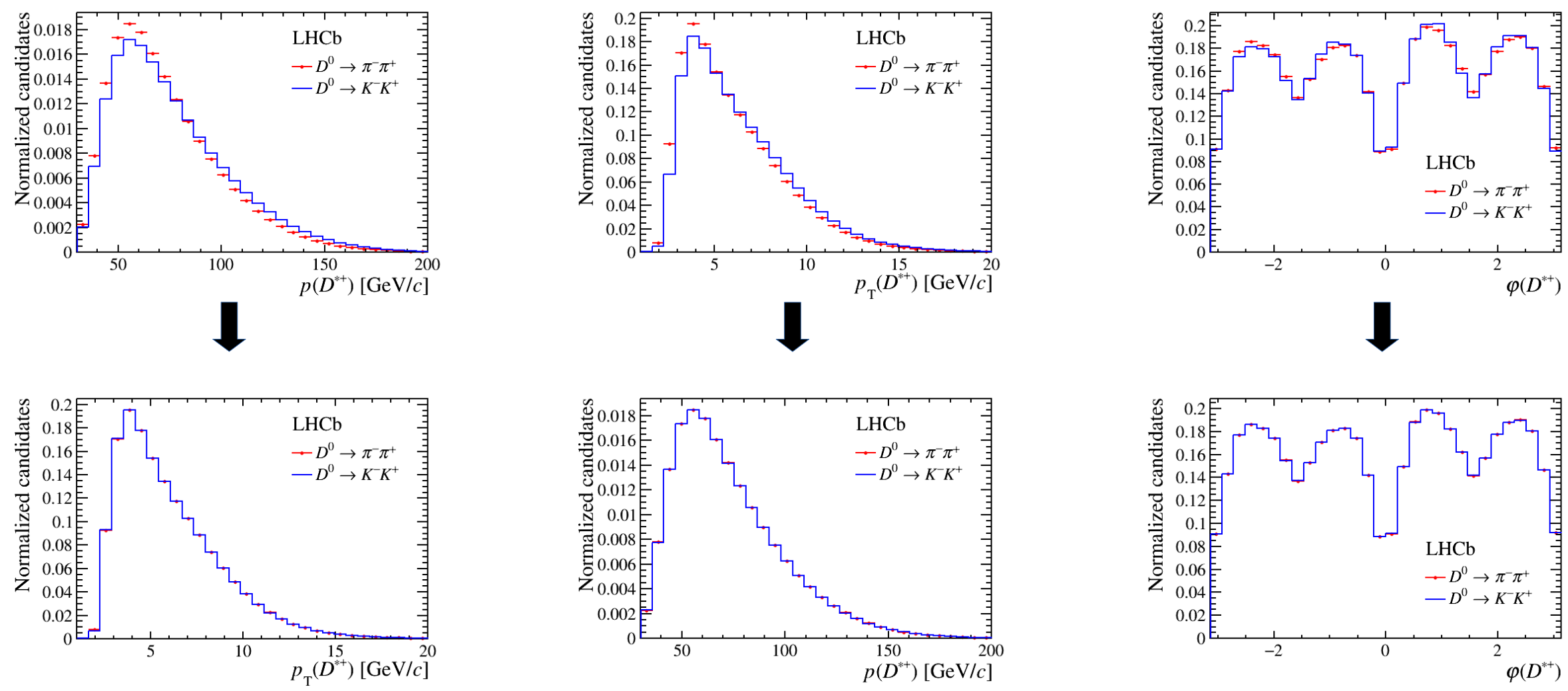

PHYS. REV. LETT. 122 (2019) 211803 


\section{CPV in Charm: systematic}

$\rightarrow$ Prompt mode dominated by:

- Fit model

- Default model: Sum of three Gaussian and Johnson Su function (prompt) and two Gaussians convolved with a power-law function (sl)

- Alternative: Fitting pseudoexperiments with alternative models

- Misreconstructed background

$\rightarrow$ Semileptonic mode dominated by mistagging of muon

- Evaluated using control sample $\mathrm{B} \rightarrow \mathrm{D}^{0}\left(\rightarrow \mathrm{K}^{-} \pi^{+}\right) \mu \mathrm{X}$

\begin{tabular}{lcc}
\hline \hline Source & $\pi$-tagged $\left[10^{-4}\right]$ & $\mu$-tagged $\left[10^{-4}\right]$ \\
\hline Fit model & 0.6 & 2 \\
Mistag & - & 4 \\
Weighting & 0.2 & 1 \\
Secondary decays & 0.3 & - \\
$B^{0}$ fraction & - & 1 \\
$B$ reco. efficiency & - & 2 \\
Peaking background & 0.5 & - \\
\hline Total & 0.9 & 5 \\
\hline \hline
\end{tabular}




\section{CPV in Charm: results}

$\rightarrow$ Run II results:

$$
\begin{gathered}
\Delta A_{C P}^{\text {prompt }}=[-18.2 \pm 3.2(\text { stat }) \pm 0.9(\text { syst })] \times 10^{-4} \\
\Delta A_{C P}^{\text {semileptonic }}=[-9 \pm 8(\text { stat }) \pm 5(\text { syst })] \times 10^{-4}
\end{gathered}
$$

$\rightarrow$ Compatible with the previous LHCb results and the world average values

$\rightarrow$ When combined with Run I LHCb results:

$$
\Delta A_{C P}^{\mathrm{RunI}+\mathrm{RunII}}=[-15.4 \pm 2.9] \times 10^{-4}
$$

$\rightarrow \underline{C P \text { violation at } 5.3 \sigma \text { level }}$

$\rightarrow \triangle A_{C P}$ is mostly sensitive to direct $C P V$ 


\section{CPV in Charm: world average}

$\rightarrow$ Updated HFLAV fit

$$
\begin{aligned}
& \Delta a_{C P}^{d i r}=(-16.4 \pm 2.8) \times 10^{-4} \\
& \Delta a_{C P}^{i n d}=(2.8 \pm 2.6) \times 10^{-4}
\end{aligned}
$$

$\rightarrow$ Compatible with SM

- Most predictions on

$$
10^{-4} \text { - } 10^{-3} \text { level }
$$

$\rightarrow$ Progress in theory calculations needed

$\rightarrow$ Observation in other channels could provide a confirmation of this effect

$\rightarrow$ Thorough study needs to be done to decide if SM or BSM

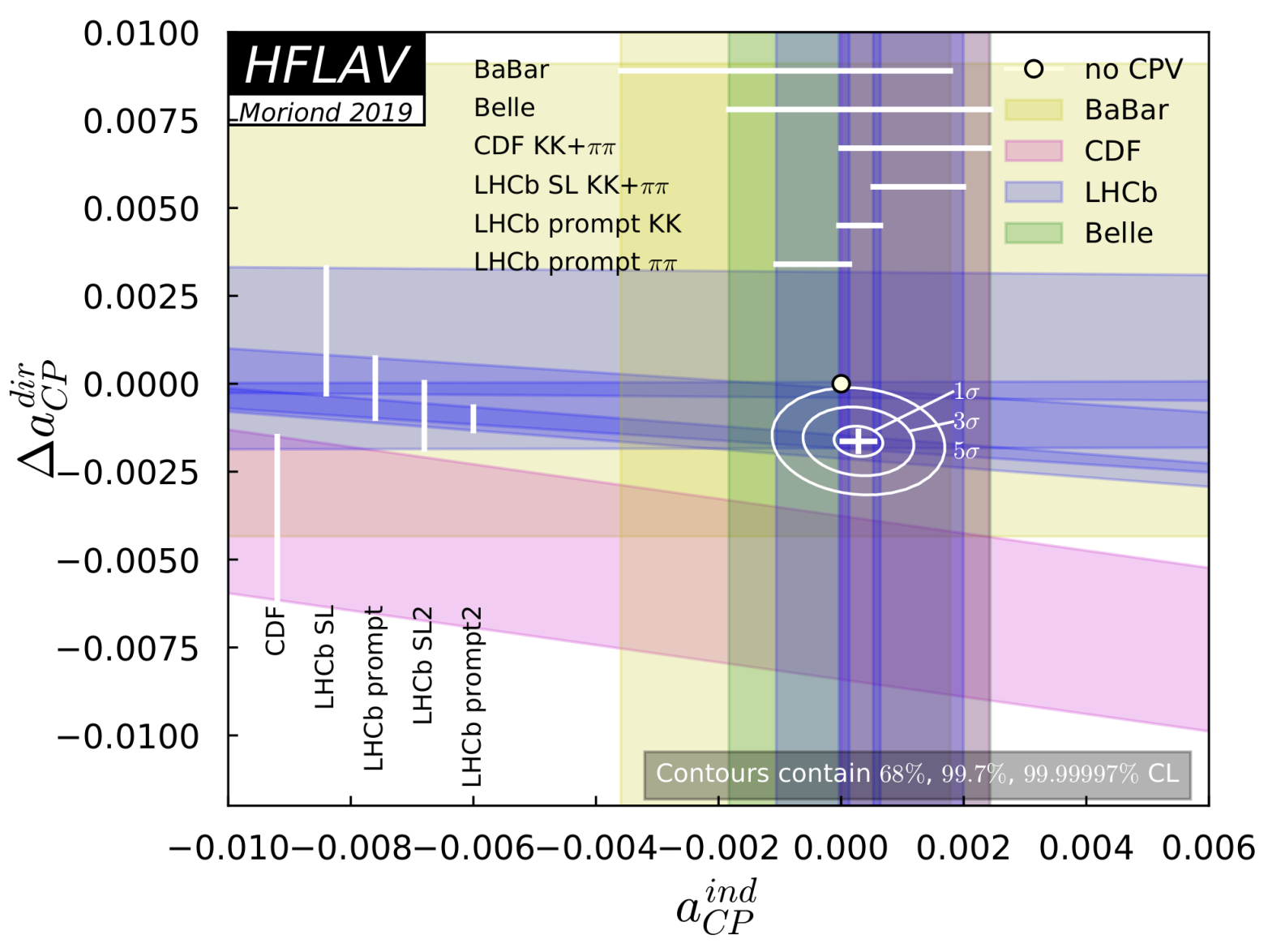
effect

$\rightarrow$ Indirect CPV still missing 


\section{Conclusion and Outlook}

$\rightarrow$ Different mass between $C P$-even and $C P$-odd $D^{0}$ states

$\rightarrow$ Direct CPV in Charm observed for the first time

$\rightarrow$ Inconclusive if SM or BSM effects

$\rightarrow$ Indirect CPV still unobserved

\section{Future prospects}

$\rightarrow$ LHCb has access to the world largest Charm sample - analyses now have to exploit it

$\rightarrow$ Belle-Il is now preparing for data taking

$\rightarrow$ Ongoing LHCb Upgrade - 5x higher luminosity and new software trigger

$\rightarrow 50 / f b$ will be collected by 2030

$\rightarrow$ Expected statistical uncertainty: $\mathcal{O}\left(10^{-4}\right)$

$\rightarrow$ Also a possibilities to utilize rare and multi-body decays 


\section{Thank you for your attention}




\section{BACKUP Slides}




\section{Planned LHCb upgrades}




\section{LHCb upgrade Phase I (Run III)}
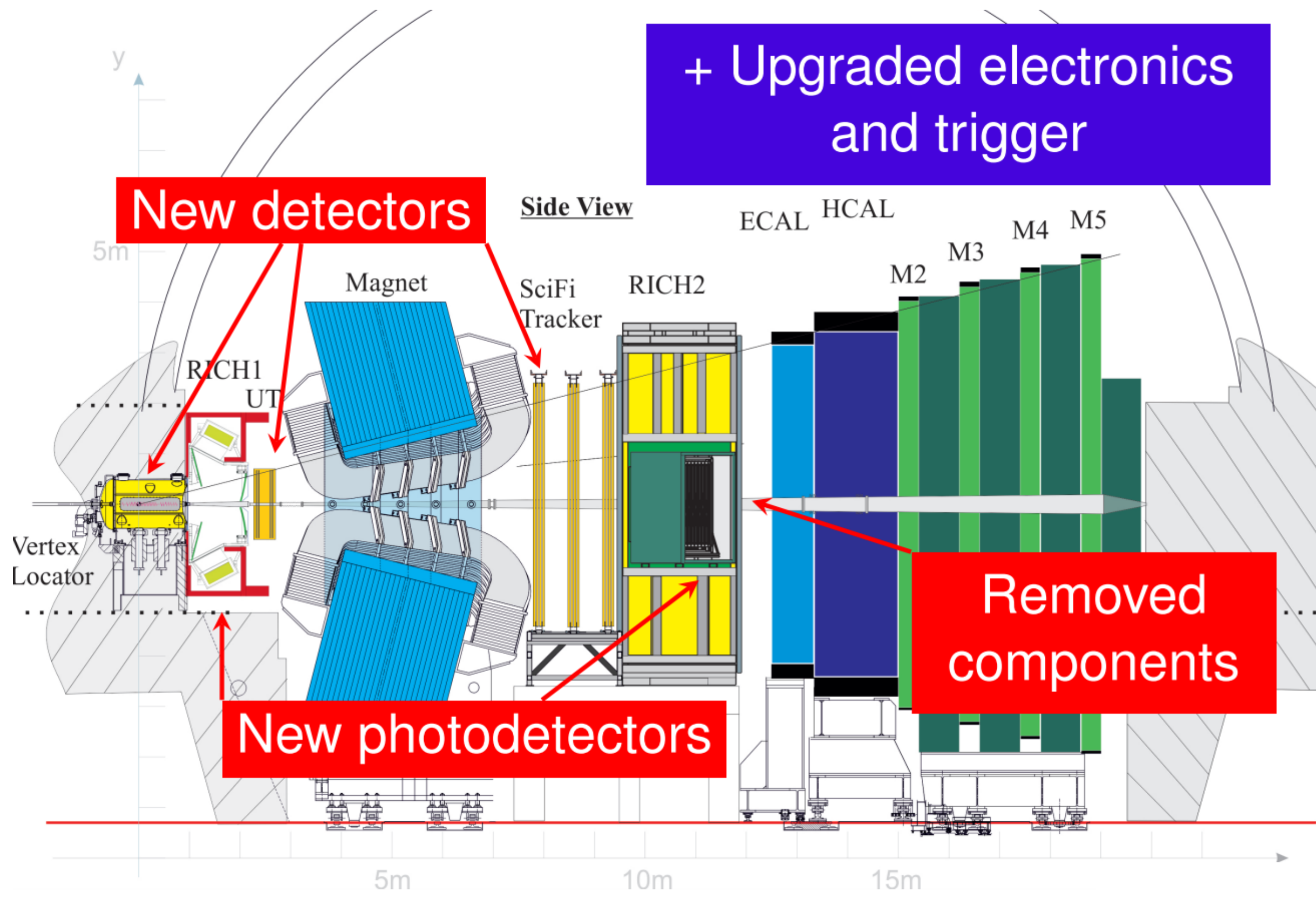


\section{LHCb upgrade Phase I (Run III)}

\begin{tabular}{|c|c|c|c|c|c|}
\hline Type & Observable & $\begin{array}{l}\text { Current } \\
\text { precision }\end{array}$ & $\begin{array}{c}\text { LHCb } \\
2018\end{array}$ & $\begin{array}{l}\text { Upgrade } \\
\left(50 \mathrm{fb}^{-1}\right)\end{array}$ & $\begin{array}{c}\text { Theory } \\
\text { uncertainty }\end{array}$ \\
\hline \multirow[t]{3}{*}{$B_{s}^{0}$ mixing } & $2 \beta_{s}\left(B_{s}^{0} \rightarrow J / \psi \phi\right)$ & $0.10[9]$ & 0.025 & 0.008 & $\sim 0.003$ \\
\hline & $2 \beta_{s}\left(B_{s}^{0} \rightarrow J / \psi f_{0}(980)\right)$ & $0.17[10]$ & 0.045 & 0.014 & $\sim 0.01$ \\
\hline & $A_{\mathrm{fs}}\left(B_{s}^{0}\right)$ & $6.4 \times 10^{-3}[18]$ & $0.6 \times 10^{-3}$ & $0.2 \times 10^{-3}$ & $0.03 \times 10^{-3}$ \\
\hline Gluonic & $2 \beta_{s}^{\mathrm{eff}}\left(B_{s}^{0} \rightarrow \phi \phi\right)$ & - & 0.17 & 0.03 & 0.02 \\
\hline \multirow{2}{*}{ penguin } & $2 \beta_{s}^{\mathrm{eff}}\left(B_{s}^{0} \rightarrow K^{* 0} \bar{K}^{* 0}\right)$ & - & 0.13 & 0.02 & $<0.02$ \\
\hline & $2 \beta^{\text {eff }}\left(B^{0} \rightarrow \phi K_{S}^{0}\right)$ & $0.17[18]$ & 0.30 & 0.05 & 0.02 \\
\hline \multirow{2}{*}{$\begin{array}{l}\text { Right-handed } \\
\text { currents }\end{array}$} & $2 \beta_{s}^{\mathrm{eff}}\left(B_{s}^{0} \rightarrow \phi \gamma\right)$ & - & 0.09 & 0.02 & $<0.01$ \\
\hline & $\tau^{\mathrm{eff}}\left(B_{s}^{0} \rightarrow \phi \gamma\right) / \tau_{B_{s}^{0}}$ & - & $5 \%$ & $1 \%$ & $0.2 \%$ \\
\hline \multirow{4}{*}{$\begin{array}{c}\text { Electroweak } \\
\text { penguin }\end{array}$} & $S_{3}\left(B^{0} \rightarrow K^{* 0} \mu^{+} \mu^{-} ; 1<q^{2}<6 \mathrm{GeV}^{2} / c^{4}\right)$ & 0.08 [14] & 0.025 & 0.008 & 0.02 \\
\hline & $s_{0} A_{\mathrm{FB}}\left(B^{0} \rightarrow K^{* 0} \mu^{+} \mu^{-}\right)$ & $25 \%[14]$ & $6 \%$ & $2 \%$ & $7 \%$ \\
\hline & $A_{\mathrm{I}}\left(K \mu^{+} \mu^{-} ; 1<q^{2}<6 \mathrm{GeV}^{2} / c^{4}\right)$ & $0.25[15]$ & 0.08 & 0.025 & $\sim 0.02$ \\
\hline & $\mathcal{B}\left(B^{+} \rightarrow \pi^{+} \mu^{+} \mu^{-}\right) / \mathcal{B}\left(B^{+} \rightarrow K^{+} \mu^{+} \mu^{-}\right)$ & $25 \%[16]$ & $8 \%$ & $2.5 \%$ & $\sim 10 \%$ \\
\hline Higgs & $\mathcal{B}\left(B_{s}^{0} \rightarrow \mu^{+} \mu^{-}\right)$ & $1.5 \times 10^{-9}[2]$ & $0.5 \times 10^{-9}$ & $0.15 \times 10^{-9}$ & $0.3 \times 10^{-9}$ \\
\hline penguin & $\mathcal{B}\left(B^{0} \rightarrow \mu^{+} \mu^{-}\right) / \mathcal{B}\left(B_{s}^{0} \rightarrow \mu^{+} \mu^{-}\right)$ & - & $\sim 100 \%$ & $\sim 35 \%$ & $\sim 5 \%$ \\
\hline Unitarity & $\gamma\left(B \rightarrow D^{(*)} K^{(*)}\right)$ & $\sim 10-12^{\circ}[19,20]$ & $4^{\circ}$ & $0.9^{\circ}$ & negligible \\
\hline triangle & $\gamma\left(B_{s}^{0} \rightarrow D_{s} K\right)$ & - & $11^{\circ}$ & $2.0^{\circ}$ & negligible \\
\hline angles & $\beta\left(B^{0} \rightarrow J / \psi K_{S}^{0}\right)$ & $0.8^{\circ}[18]$ & $0.6^{\circ}$ & $0.2^{\circ}$ & negligible \\
\hline Charm & $A_{\Gamma}$ & $2.3 \times 10^{-3}[18]$ & $0.40 \times 10^{-3}$ & $0.07 \times 10^{-3}$ & - \\
\hline$C P$ violation & $\Delta A_{C P}$ & $2.1 \times 10^{-3}[5]$ & $0.65 \times 10^{-3}$ & $0.12 \times 10^{-3}$ & - \\
\hline
\end{tabular}

CERN/LHCC 2012-007 


\section{LHCb upgrade Phase II (Run V)}

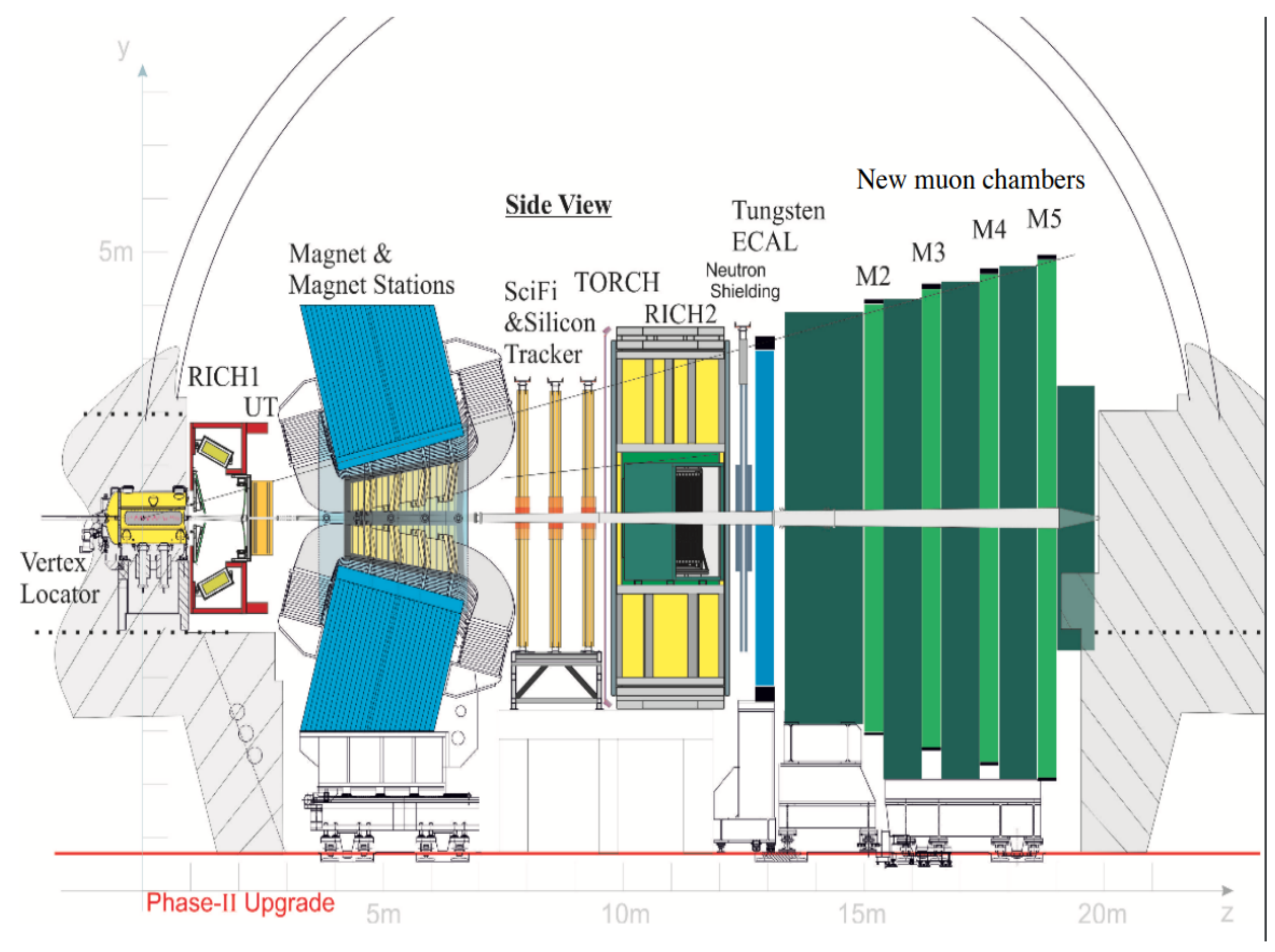




\section{LHCb upgrade Phase II (Run V)}

\begin{tabular}{|c|c|c|}
\hline Topics and observables & Experimental reach & Remarks \\
\hline \multicolumn{3}{|l|}{ EW Penguins } \\
\hline 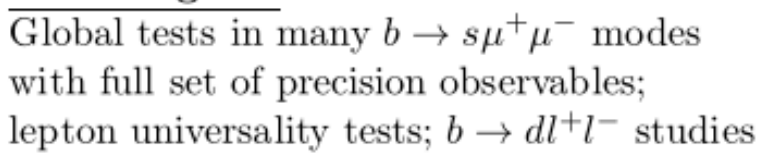 & $\begin{array}{l}\text { e.g. } 440 \mathrm{k} B^{0} \rightarrow K^{*} \mu^{+} \mu^{-} \& 70 \mathrm{k} \Lambda_{b}^{0} \rightarrow \Lambda \mu^{+} \mu^{-} \\
\text {Phase-II } b \rightarrow d \mu^{+} \mu^{-} \approx \text { Run-1 } b \rightarrow s \mu^{+} \mu^{-} \\
\text {sensitivity. }\end{array}$ & $\begin{array}{l}\text { Phase-II ECAL required for } \\
\text { lepton universality tests. }\end{array}$ \\
\hline \multicolumn{3}{|l|}{ Photon polarisation } \\
\hline 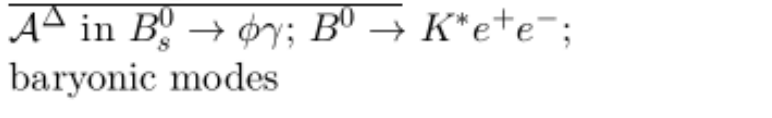 & $\begin{array}{l}\text { Uncertainty on } \mathcal{A}^{\Delta} \approx 0.02 \\
\sim 10 k \Lambda_{b}^{0} \rightarrow \Lambda \gamma, \Xi_{b} \rightarrow \Xi \gamma, \Omega_{b}^{-} \rightarrow \Omega \gamma\end{array}$ & $\begin{array}{l}\text { Strongly dependent on } \\
\text { performance of ECAL. }\end{array}$ \\
\hline \multicolumn{3}{|l|}{$b \rightarrow c l^{-} \overline{\nu_{l}}$ lepton-universality tests } \\
\hline $\begin{array}{l}\text { Polarisation studies with } B \rightarrow D^{(*)} \tau^{-} \bar{\nu}_{\tau} ; \\
\tau^{-} / \mu^{-} \text {ratios with } B_{s}^{0}, A_{b}^{0} \text { and } B_{c}^{+} \text {modes } \\
\boldsymbol{B}_{\boldsymbol{s}}^{\mathbf{0}}, \boldsymbol{B}^{\mathbf{0}} \rightarrow \boldsymbol{\mu}^{+} \boldsymbol{\mu}^{-}\end{array}$ & $\begin{array}{l}\text { e.g. } 8 \mathrm{M} B \rightarrow D^{*} \tau^{-} \overline{\nu_{\tau}}, \tau^{-} \rightarrow \mu^{-} \overline{\nu_{\mu}} \nu_{\tau} \\
\& \sim 100 k \tau^{-} \rightarrow \pi^{-} \pi^{+} \pi^{-}\left(\pi^{0}\right) \nu_{\tau}\end{array}$ & $\begin{array}{l}\text { Additional sensitivity expected } \\
\text { from low- } p \text { tracking. }\end{array}$ \\
\hline$\left.\overline{R \equiv \mathcal{B}\left(B^{0} \rightarrow \mu^{+}\right.} \mu^{-}\right) / \mathcal{B}\left(B_{s}^{0} \rightarrow \mu^{+} \mu^{-}\right)$ & Uncertainty on $R \approx 20 \%$ & \\
\hline$\tau_{B_{s}^{0} \rightarrow \mu^{+} \mu^{-}} ; C P$ asymmetry & Uncertainty on $\tau_{B_{s}^{0} \rightarrow \mu^{+} \mu^{-}} \approx 0.03 \mathrm{ps}$ & \\
\hline \multicolumn{3}{|l|}{ LFV $\tau$ decays } \\
\hline $\begin{array}{l}\overline{\tau^{-} \rightarrow \mu^{+} \mu^{-} \mu^{-}}, \tau^{-} \rightarrow h^{+} \mu^{-} \mu^{-} \\
\tau^{-} \rightarrow \phi \mu^{-}\end{array}$ & Sensitive to $\tau^{-} \rightarrow \mu^{+} \mu^{-} \mu^{-}$at $10^{-9}$ & $\begin{array}{l}\text { Phase-II ECAL valuable } \\
\text { for background suppression. }\end{array}$ \\
\hline \multicolumn{3}{|l|}{ CKM tests } \\
\hline $\begin{array}{l}\gamma \text { with } B^{-} \rightarrow D K^{-}, B_{s}^{0} \rightarrow D_{s}^{+} K^{-} \text {etc. } \\
\phi_{s} \text { with } B_{s}^{0} \rightarrow J / \psi K^{+} K^{-}, J / \psi \pi^{+} \pi^{-} \\
\phi_{s}^{s \bar{s}} \text { with } B_{s}^{0} \rightarrow \phi \phi \\
\Delta \Gamma_{d} / \Gamma_{d} \\
\text { Semileptonic asymmetries } a_{\text {sl }}^{d, s} \\
\left|V_{u b}\right| /\left|V_{c b}\right| \text { with } \Lambda_{b}^{0}, B_{s}^{0} \text { and } B_{c}^{+} \text {modes }\end{array}$ & $\begin{array}{l}\text { Uncertainty on } \gamma \approx 0.4^{\circ} \\
\text { Uncertainty on } \phi_{s} \approx 3 \mathrm{mrad} \\
\text { Uncertainty on } \phi_{s}^{s \bar{s} s} \approx 8 \mathrm{mrad} \\
\text { Uncertainty on } \Delta \Gamma_{d} / \Gamma_{d} \sim 10^{-3} \\
\text { Uncertainties on } a_{\mathrm{sl}}^{d, s} \sim 10^{-4} \\
\text { e.g. } 120 k B_{c}^{+} \rightarrow D^{0} \mu^{-} \overline{\nu_{\mu}}\end{array}$ & $\begin{array}{l}\text { Additional sensitivity expected } \\
\text { in } C P \text { observables from Phase-II } \\
\text { ECAL and low- } p \text { tracking. } \\
\text { Approach SM value. } \\
\text { Approach SM value for } a_{\mathrm{sl}}^{d} \text {. } \\
\text { Significant gains achievable from } \\
\text { thinning or removing RF-foil. }\end{array}$ \\
\hline \multicolumn{3}{|l|}{ Charm } \\
\hline $\begin{array}{l}C P \text {-violation studies with } D^{0} \rightarrow h^{+} h^{-}, \\
D^{0} \rightarrow K_{\mathrm{S}}^{0} \pi^{+} \pi^{-} \text {and } D^{0} \rightarrow K^{\mp} \pi^{ \pm} \pi^{+} \pi^{-}\end{array}$ & $\begin{array}{l}\text { e.g. } 4 \times 10^{9} D^{0} \rightarrow K^{+} K^{-} \\
\text {Uncertainty on } A_{\Gamma} \sim 10^{-5}\end{array}$ & Access $C P$ violation at $\mathrm{SM}$ values. \\
\hline \multicolumn{3}{|l|}{ Strange } \\
\hline$\overline{\text { Rare decay searches }}$ & Sensitive to $K_{\mathrm{S}}^{0} \rightarrow \mu^{+} \mu^{-}$at $10^{-12}$ & $\begin{array}{l}\text { Additional sensitivity possible with } \\
\text { downstream trigger enhancements. }\end{array}$ \\
\hline
\end{tabular}




\section{LHC timeline}

LHC roadmap: according to MTP 2016-2020 V1

LS2 starting in 2019

LS3 LHC: starting in 2024 Injectors: in 2025
$=>24$ months +3 months $B C$

$=>30$ months +3 months BC $\Rightarrow 13$ months +3 months BC
Physics

Shutdown

Beam commissioning

Technical stop

\begin{tabular}{|c|c|c|c|c|c|c|c|c|c|c|c|c|c|c|c|c|c|c|c|c|c|c|c|c|c|}
\hline \multicolumn{3}{|c|}{2015} & \multicolumn{4}{|c|}{2016} & \multicolumn{4}{|c|}{2017} & \multicolumn{4}{|c|}{2018} & \multicolumn{4}{|c|}{2019} & \multicolumn{4}{|c|}{2020} & \multicolumn{3}{|c|}{2021} \\
\hline \begin{tabular}{l|l}
$Q 1$ & $Q$
\end{tabular} & Q3 & Q4 & Q1 & $Q 2$ & Q3 & Q4 & Q1 & Q2 & Q3 & Q4 & Q1 & Q2 & Q3 & Q4 & Q1 & Q2 & Q3 & Q4 & Q1 & Q2 & Q3 & Q4 & Q1 & Q2:Q3 & 124 \\
\hline
\end{tabular}

LHC

Injectors

Run 2

LS 2

PHASE 1-

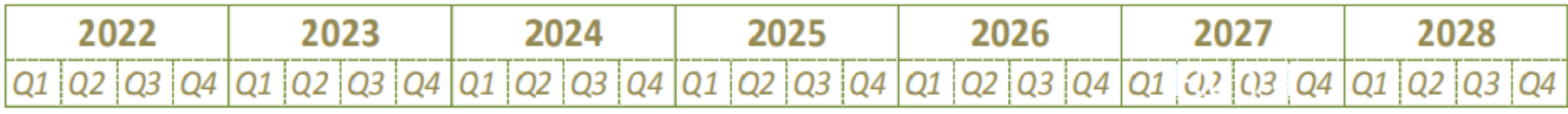

LHC

Injectors

Run 3

LS 3

Run 4

PHASE 2

\begin{tabular}{|c|c|c|c|c|c|c|c|c|c|c|c|c|c|c|c|c|c|c|c|c|c|c|c|c|c|}
\hline \multicolumn{3}{|c|}{2029} & \multicolumn{4}{|c|}{2030} & \multicolumn{4}{|c|}{2031} & \multicolumn{4}{|c|}{2032} & \multicolumn{4}{|c|}{2033} & \multicolumn{4}{|c|}{2034} & \multicolumn{3}{|c|}{2035} \\
\hline Q1 & $\begin{array}{l:l} & \\
\end{array}$ & Q4 & Q1 & $Q 2$ & Q3 & Q4 & Q1 & Q2 & Q3 & Q4 & $Q$ & Q2 & Q3 & Q4 & Q1 & Q2 & (19) & 124 & Q1 & Q2 & Q3 & Q4 & Q1 & Q2 $Q$ & $\begin{array}{l}3 \\
\end{array}$ \\
\hline
\end{tabular}

LHC

Injectors

LS 4

Run 5

LS 5 


\section{$D^{0}$ mass eigenstates $\Delta \mathrm{m}$ : fits}

$\rightarrow$ Simultaneous least-squares fit* for prompt and semileptonic data

$\rightarrow$ Offset due to sample-specific efficiency variations across Dalitz plot

$\chi^{2}=\sum_{\operatorname{Pr}, \mathrm{SL}} \sum_{\mathrm{LL}, \mathrm{DD}} \sum_{b, j}\left[\frac{\left(N_{-b j}^{+}-N_{b j}^{+} R_{b j}^{+}\right)^{2}}{\left(\sigma_{-b j}^{+}\right)^{2}+\left(\sigma_{b j}^{+} R_{b j}^{+}\right)^{2}}+\frac{\left(N_{-b j}^{-}-N_{b j}^{-} R_{b j}^{-}\right)^{2}}{\left(\sigma_{-b j}^{-}\right)^{2}+\left(\sigma_{b j}^{-} R_{b j}^{-}\right)^{2}}\right]+\chi_{X}^{2}$,

$\chi_{X}^{2}=\sum_{a, b}\left[X_{a}^{\mathrm{CLEO}}-X_{a}\right]\left(V_{\mathrm{CLEO}}^{-1}\right)_{a b}\left[X_{b}^{\mathrm{CLEO}}-X_{b}\right]$.

$\rightarrow$ Simultaneously applied for prompt/semileptonic data, D\%anti-D ${ }^{0}$

$\rightarrow$ Two fits: CP symmetry scenario and indirect CPV allowed 


\section{Future prospects for Run III and beyond}




\section{Prospect for indirect CPV searches}

$\rightarrow$ Results on the indirect CPV is already dominated by $\mathrm{LHCb}$

\begin{tabular}{|c|c|c|c|c|}
\hline & $\sigma(x)\left[10^{-3}\right]$ & $\sigma(y)\left[10^{-3}\right]$ & $\sigma(q / p)\left[10^{-3}\right]$ & $\sigma(\phi)[\mathrm{mrad}]$ \\
\hline HFAG 2016 & 1.4 & 0.7 & 80 & 173 \\
\hline Run II & 0.8 & 0.6 & 47 & 83 \\
\hline Run III & 0.3 & 0.2 & 17 & 32 \\
\hline Belle II $\left(50 \mathrm{ab}^{-1}\right)^{1}$ & 0.8 & 0.5 & 60 & 70 \\
\hline
\end{tabular}

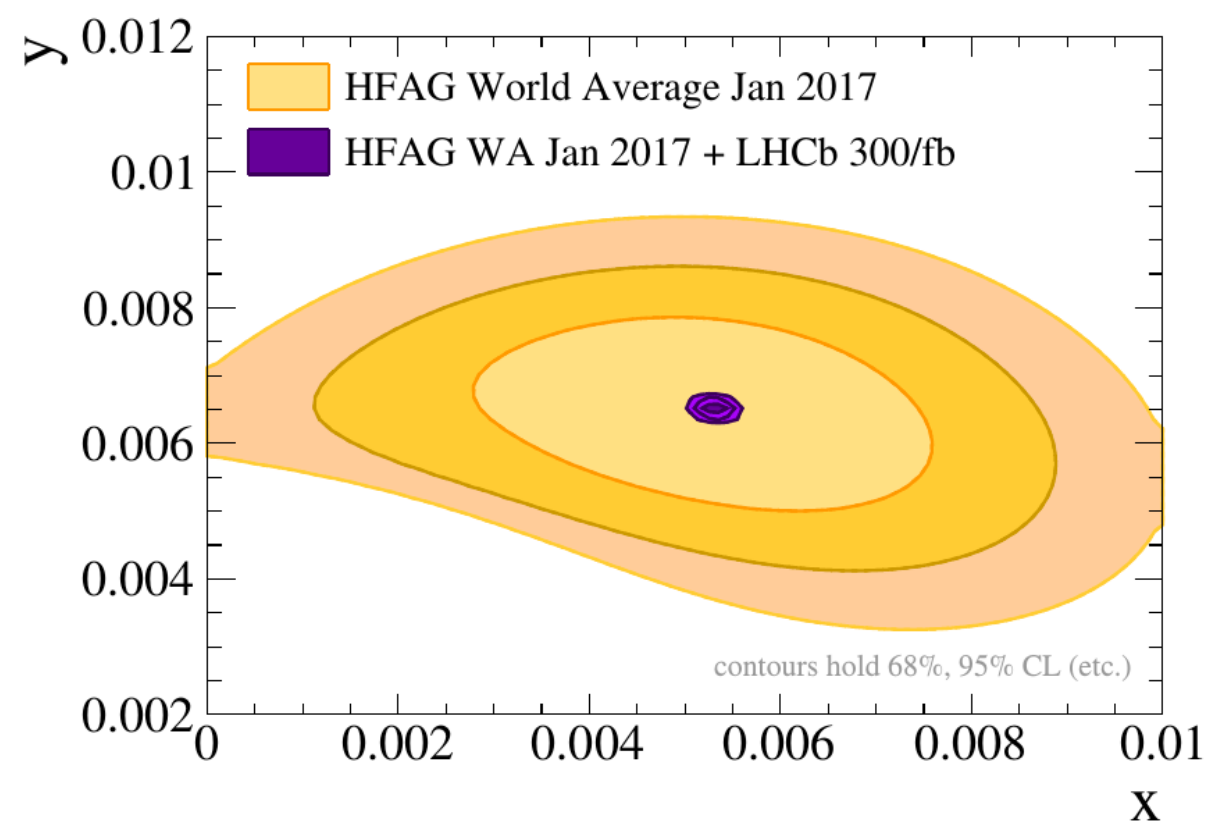

$\mathrm{X}$

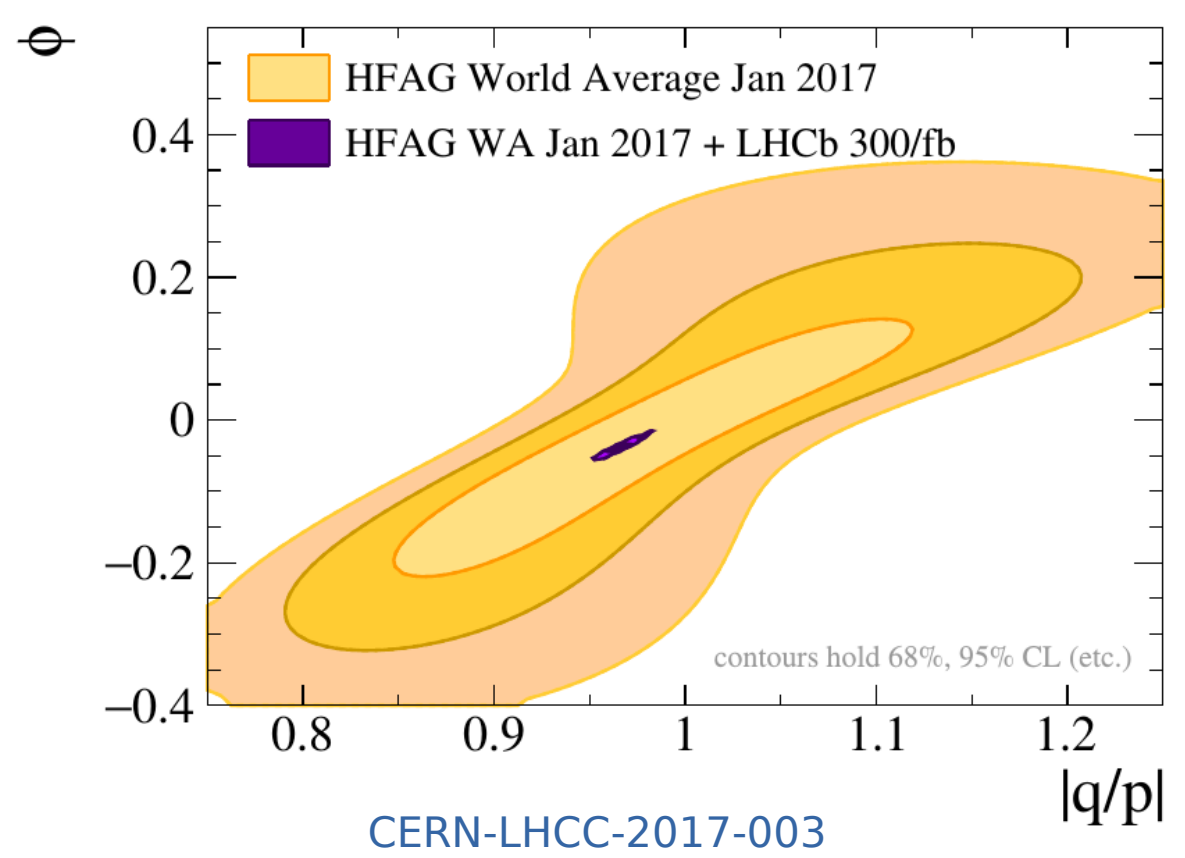




\section{Prospects for direct CPV searches}

$\rightarrow$ Precision is already at $\mathcal{O}\left(10^{-3}\right)$ level, one evidence for CPV in charm

$\rightarrow$ With the the Run III data (50 $\mathrm{fb}^{-1}$ in combination with Run I+II) the precision will be comparable with the SM prediction at $\mathcal{S}\left(10^{-4}\right)$ level

$\rightarrow$ Need for precise BR input by Belle II/HIEPA: $\mathrm{D}^{0} \rightarrow \pi^{0} \pi^{0}, \mathrm{D}^{0} \rightarrow \mathrm{K}_{\mathrm{S}} \mathrm{K}_{\mathrm{S}}, \mathrm{D}^{0} \rightarrow \pi^{0} \pi^{+}$

1) Multibody decays [slide: 42-43]

2) Rare decays (radiative, leptonic) [slide: 45]

3) Double Cabibbo Suppressed (DCS) decays (e.g. $\mathrm{D}^{+} \rightarrow \mathrm{K}^{+} \pi^{+} \pi^{-} / \mathrm{K}^{+} \mathrm{K}^{-} \mathrm{K}^{+}$)

4) Exploring charm baryons [slide: 46-47]

$\rightarrow$ Measured $1^{\text {st }}$ evidence for CPV in baryons: $\Lambda_{\mathrm{b}} \rightarrow \mathrm{p} 3 \pi \quad$ [Nature Phys. 13, 391-396 (2017)] 


\section{Prospect: $C P V$ in $\mathbf{N}$-body decays}

$\rightarrow$ Strong phase vary in Phase Space $\rightarrow$ this leads to local CPV asymmetries

$\rightarrow$ Need for detailed study of Phase space

$\rightarrow$ Model dependent: amplitude analysis

$\rightarrow$ Model independent approach:

$\rightarrow \mathrm{S}_{\mathrm{cp}}$ approach

Binned approach

$\rightarrow$ Significance of asymmetry in Dalitz plot

[PLB 728585 (2014)]

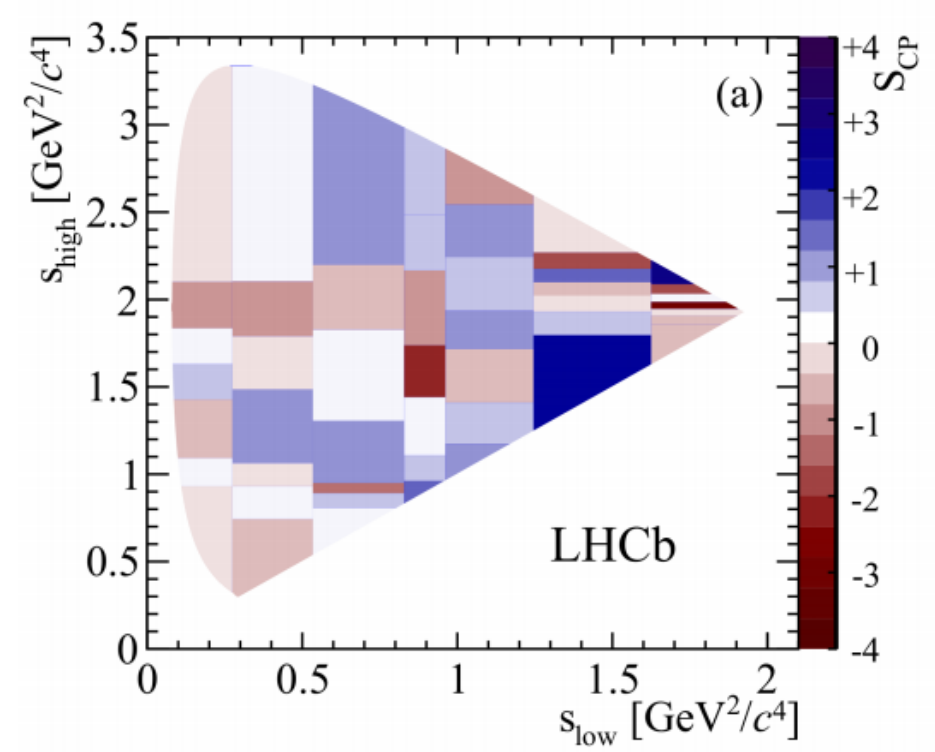

Unbinned approach (Energy test)

$\rightarrow$ Testing data consistency with no-CPV hypothesis

$\rightarrow$ Significance of asymmetry for each event

[PLB 740158 (2015)]

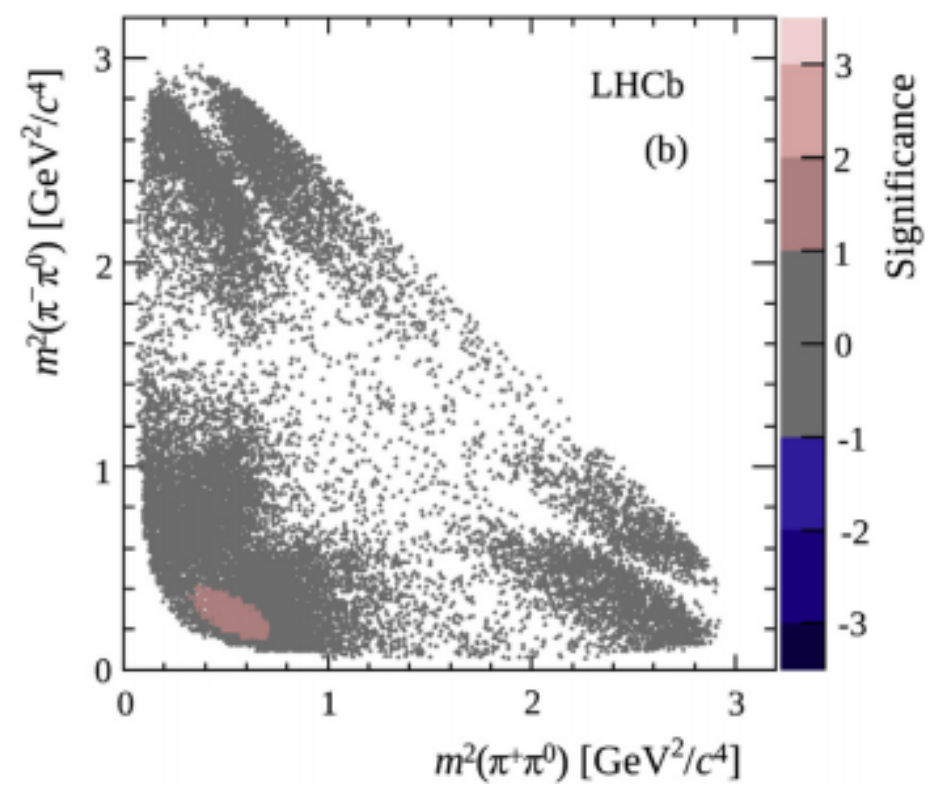




\section{Prospect: direct CPV 4-body decays}

$\rightarrow$ The more precise detector $\rightarrow$ more possibilities with the study of D multi-body decays

$\rightarrow$ The 2+3-body decays: only P-even amplitude accessible $\rightarrow$ CPV via C-violation

$\rightarrow$ The 4-body decays: also P-odd amplitudes $\rightarrow$ CPV via P-violation

$\rightarrow$ We can write: $\quad A_{C P}^{P-\text { even }} \approx \sin \Delta \phi_{\text {weak }} \sin \Delta \phi_{\text {strong }}$

$$
A_{C P}^{P-\text { odd }} \approx \sin \Delta \phi_{\text {weak }} \cos \Delta \phi_{\text {strong }}
$$

$\rightarrow$ First measurement: $\mathrm{D}^{0} \rightarrow \pi^{+} \pi^{-} \pi^{+} \pi^{-}, P$-odd CPV with the $2.7 \sigma$ significance [PLB 769 345-356 (2017)]

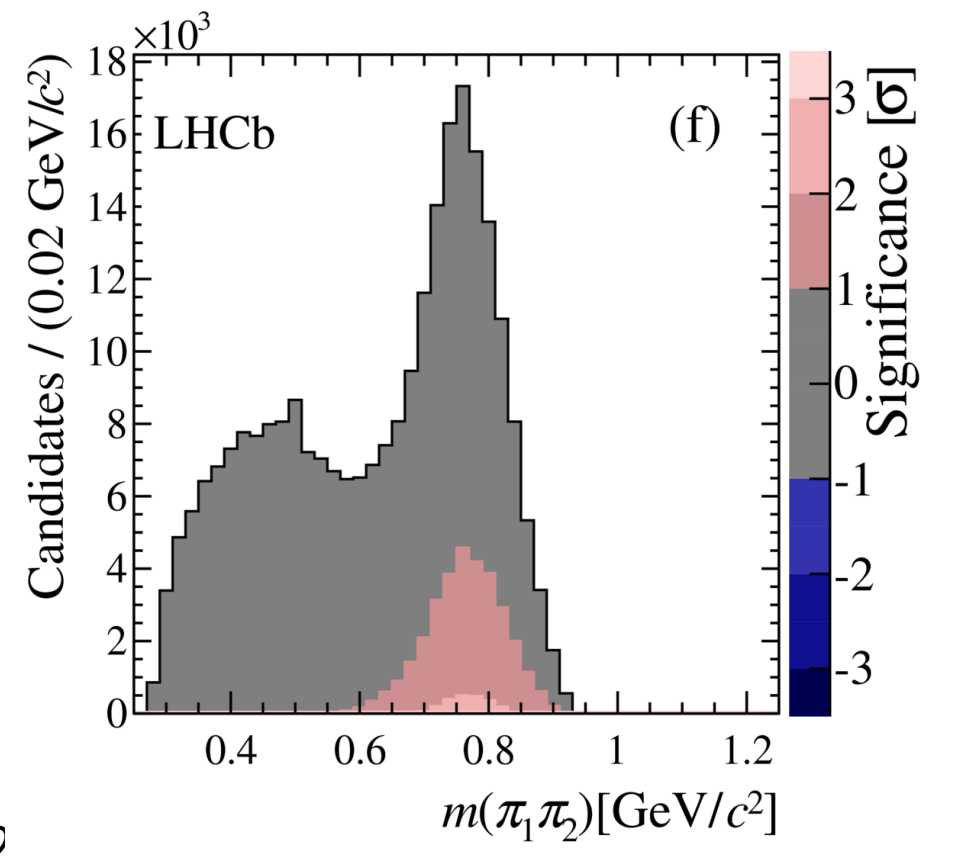

\begin{tabular}{|c|c|c|c|}
\hline \multicolumn{1}{|c|}{ Mode } & $A_{C P}^{P-\text { odd }}\left[10^{-3}\right]$ & Exp. & Ref. \\
\hline $\mathrm{D}^{+} \rightarrow \mathrm{K}_{\mathrm{S}} \mathrm{K}^{+} \pi^{+} \pi^{-}$ & $-12 \pm 10 \pm 5$ & BaBar & $\begin{array}{c}\text { PRD 84 } \\
031103\end{array}$ \\
\hline $\mathrm{D}^{0} \rightarrow \mathrm{K}^{+} \mathrm{K}^{-} \pi^{+} \pi^{-}$ & $1.8 \pm 2.9 \pm 0.4$ & LHCb & $\begin{array}{c}\text { JHEP 10 005 } \\
(2014)\end{array}$ \\
\hline $\mathrm{D}^{0} \rightarrow \mathrm{K}_{\mathrm{S}} \pi^{+} \pi^{-} \pi^{0}$ & $-0.3 \pm 1.4_{-0.8}^{+0.2}$ & Belle & $\begin{array}{c}\text { PRD 95 } \\
091101\end{array}$ \\
\hline
\end{tabular}




\section{Prospect: $C P V$ in rare decays}

$\rightarrow$ Large contribution from penguin diagrams $\rightarrow$ larger values of CPV expected

$\rightarrow$ Two main categories: Leptonic and Radiative decays

\section{Leptonic decays}

$\rightarrow$ First observation of $\mathrm{D}^{0} \rightarrow \mathrm{K}^{+} \mathrm{K}^{-} \mu^{+} \mu^{-}$ and $\mathrm{D}^{0} \rightarrow \pi^{+} \pi^{-} \mu^{+} \mu$

$\rightarrow 5.4 \sigma$ signal

$\rightarrow$ CPV up to $\mathcal{O}\left(10^{-2}\right)$

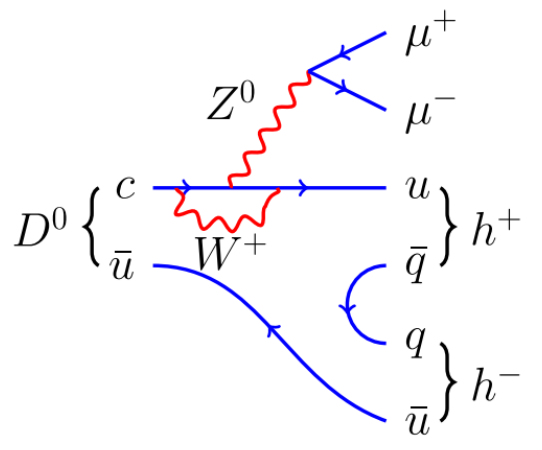

Radiative decays

$\rightarrow$ Large CPV within SM, up to $10 \%$

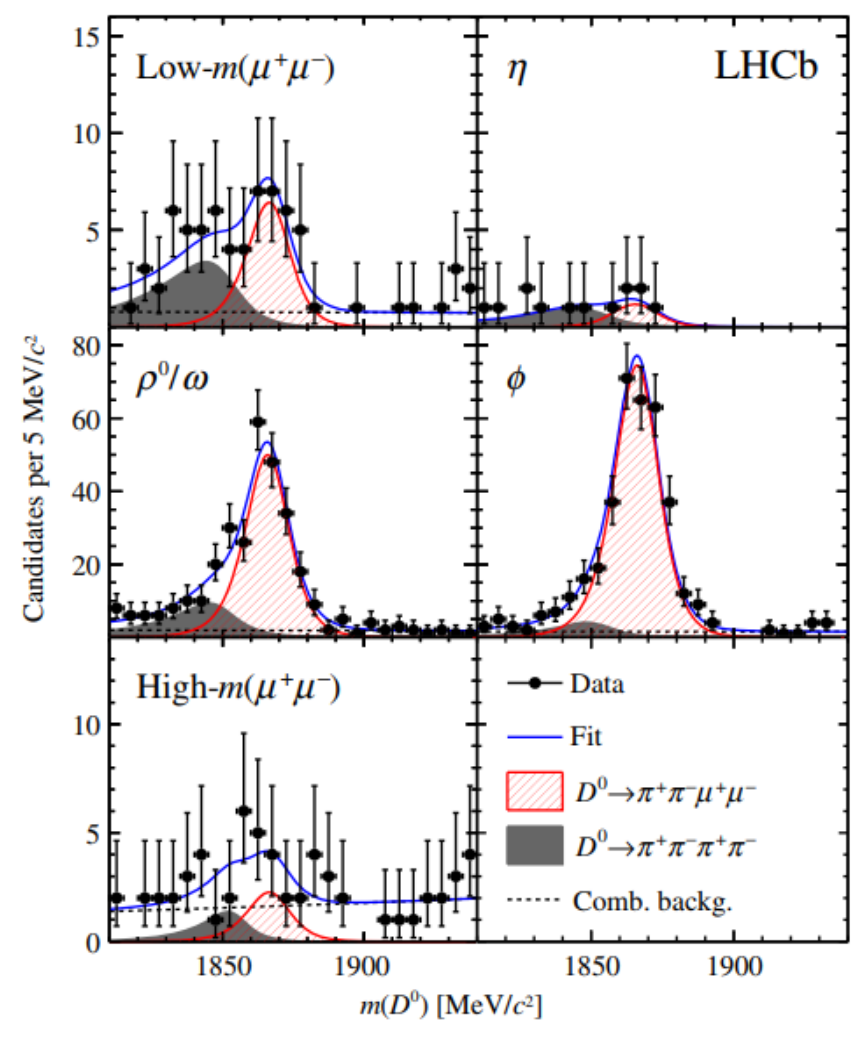

$\rightarrow$ With the upgrade, LHCb will be competitive in $\mathrm{D}^{0} \rightarrow \rho \gamma, \phi \gamma, \mathrm{K}^{*} \gamma$

$\rightarrow$ Belle measurement ${ }^{1}: A_{C P}\left(\mathrm{D}^{0} \rightarrow \rho^{0} \gamma\right)=(+5.6 \pm 15.1 \pm 0.6) \%$ 


\section{CPV in charmed baryons}

$\rightarrow$ Several theoretical works about CPV in charmed baryons

$\rightarrow$ Multibody decays are preferred due to larger BR and access to CPV-odd observables

SCS

$\rightarrow$ SM amplitudes are less suppressed, lower sensitivity to BSM amplitudes

$\rightarrow$ Suggested channels: $\Lambda_{\mathrm{c}} \rightarrow \mathrm{p} \pi^{+} \pi^{-} / \mathrm{pK}^{+} \mathrm{K}^{-}, \Xi_{\mathrm{c}}^{+} \rightarrow \mathrm{pK}^{-} \pi^{+}$

\section{DCS}

$\rightarrow$ Significant suppression of SM amplitudes

$\rightarrow$ No CP asymmetry from SM in such amplitudes

$\rightarrow$ Suggested channel: $\Lambda_{\mathrm{c}}^{+} \rightarrow \mathrm{pK}^{+} \pi^{-}$ 


\section{CPV in $\Lambda_{\mathrm{c}}^{+} \rightarrow \mathrm{pK}^{-} \mathrm{K}^{+}$and $\Lambda_{\mathrm{c}}^{+} \rightarrow \mathrm{p} \pi^{+} \pi^{-}$}

$\rightarrow$ First measurement of CPV parameters in three-body $\Lambda_{\mathrm{c}}^{+}$decays

$\rightarrow$ Full Run I $\left(3 \mathrm{fb}^{-1}\right)$ data used

$\rightarrow$ The $\Lambda_{\mathrm{b}}^{0} \rightarrow \Lambda_{\mathrm{c}}^{+} \mu^{-} \mathrm{X}$ decay channel used in order to reduce prompt background

$\rightarrow$ Two SCS decays studied: $\Lambda_{\mathrm{c}}^{+} \rightarrow \mathrm{pK}^{-} \mathrm{K}^{+}(25 \mathrm{k}) \Lambda_{\mathrm{c}}^{+} \rightarrow \mathrm{p} \pi^{-} \pi^{+}$(160 k)

$\rightarrow$ Measurement of difference $\Delta \mathcal{A}_{C P}=\mathcal{A}_{\text {raw }}\left(\mathrm{pK}^{-} \mathrm{K}^{+}\right)-\mathcal{A}_{\text {raw }}\left(\mathrm{p} \pi^{-} \pi^{+}\right)$ in order to cancel production and detection asymmetry
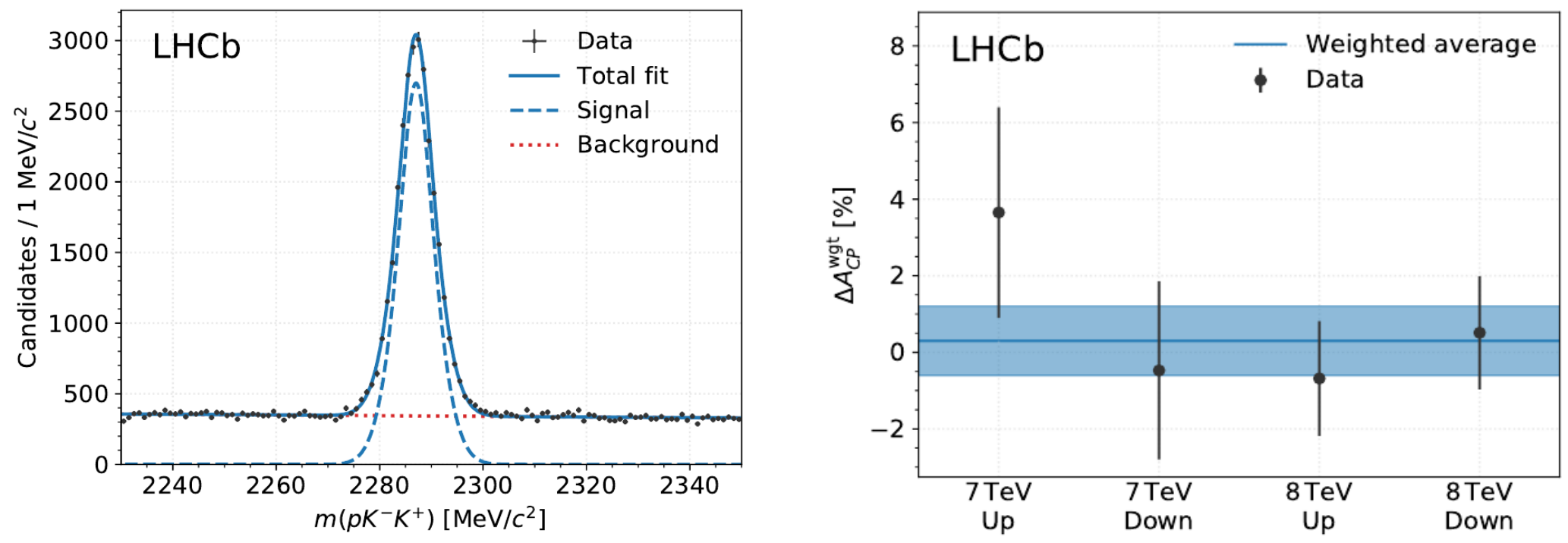

$\rightarrow$ Final result: $\Delta \mathcal{A}_{\mathbf{C P}}=(\mathbf{0 . 3 0} \pm \mathbf{0 . 9 1} \pm \mathbf{0 . 6 1}) \%$ 


\section{CPV in $\Lambda_{\mathrm{c}}^{+} \rightarrow \mathrm{pK}^{-} \mathrm{K}^{+}$and $\Lambda_{\mathrm{c}}^{+} \rightarrow \mathrm{p} \pi^{+} \pi^{-}$}

$\rightarrow$ Obtained results in the 4 bins: collision energy and magnet polarity

$\rightarrow$ First result of search for direct CPV search in three-body $\Lambda_{\mathrm{c}}^{+}$decays:

$$
\Delta \mathcal{A}_{\mathrm{CP}}=[0.30 \pm 0.91 \text { (stat.) } \pm 0.61 \text { (syst.) }] \%
$$

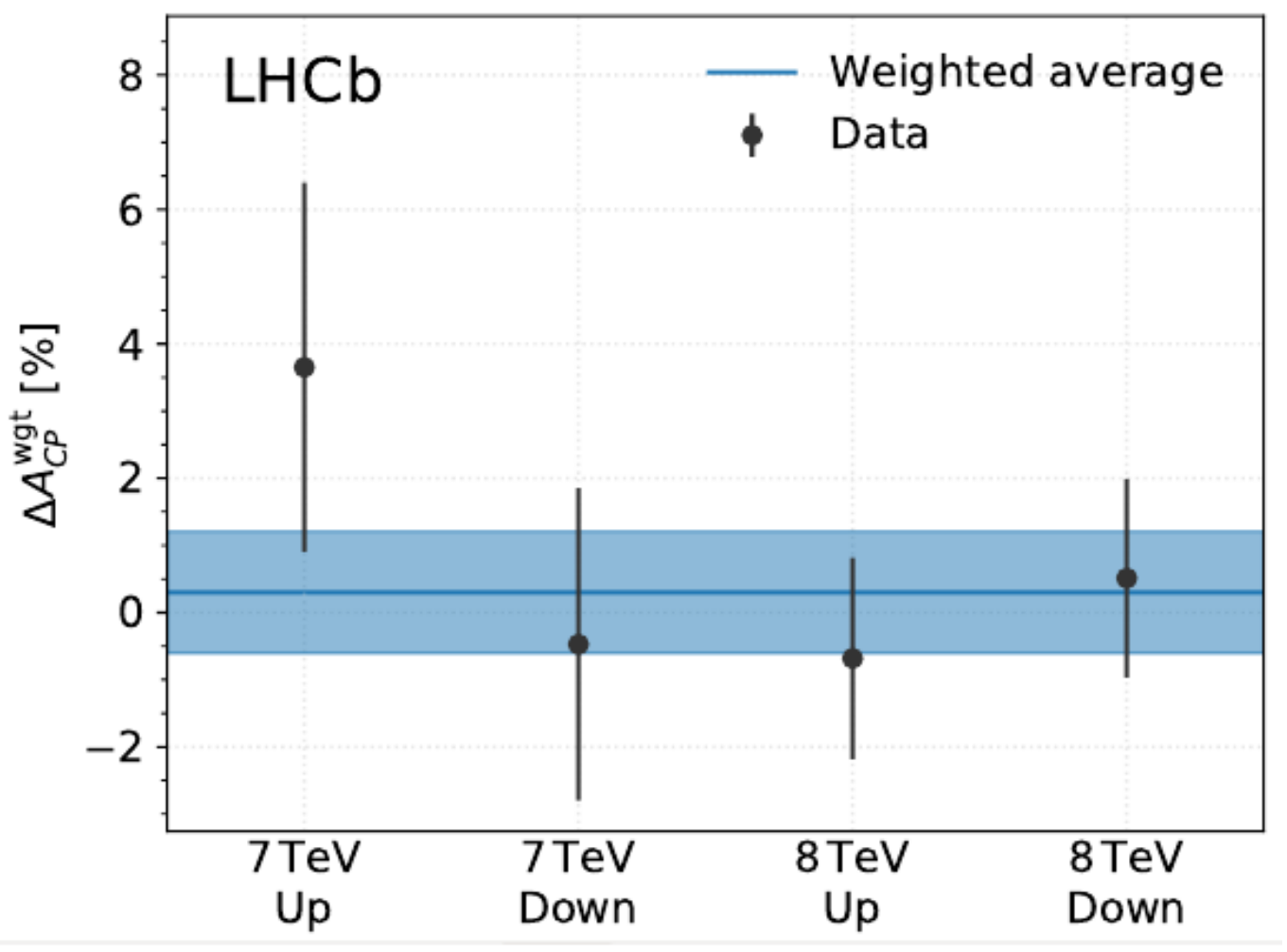

$\rightarrow$ Result shows no sign of direct CPV

$\rightarrow$ More data required for more precise measurement 